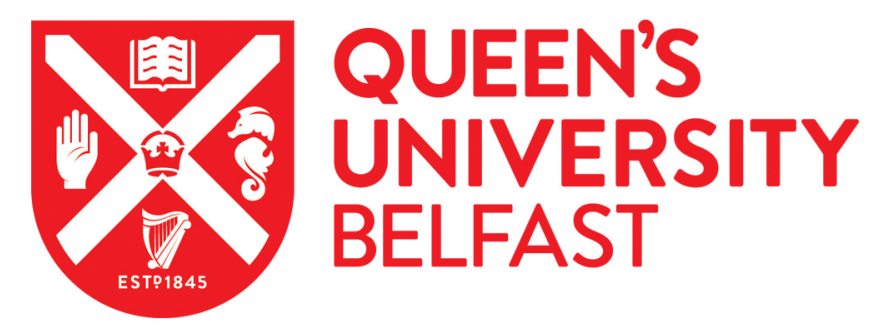

\title{
Optimum Imaging Strategies for Advanced Prostate Cancer: ASCO Guideline
}

Trabulsi, E. J., Rumble, R. B., Jadvar, H., Hope, T., Pomper, M., Turkbey, B., Rosenkrantz, A. B., Verma, S., Margolis, D. J., Froemming, A., Oto, A., Purysko, A., Milowsky, M. I., Schlemmer, H-P., Eiber, M., Morris, M. J., Choyke, P. L., Padhani, A., Oldan, J., ... Vargas, H. A. (2020). Optimum Imaging Strategies for Advanced Prostate Cancer: ASCO Guideline. Journal of Clinical Oncology : official journal of the American Society of Clinical Oncology. https://doi.org/10.1200/JCO.19.02757

Published in:

Journal of Clinical Oncology : official journal of the American Society of Clinical Oncology

\section{Document Version:}

Publisher's PDF, also known as Version of record

\section{Queen's University Belfast - Research Portal:}

Link to publication record in Queen's University Belfast Research Portal

\section{Publisher rights}

(C) 2020 by American Society of Clinical Oncology. This work is made available online in accordance with the publisher's policies. Please refer to any applicable terms of use of the publisher.

\section{General rights}

Copyright for the publications made accessible via the Queen's University Belfast Research Portal is retained by the author(s) and / or other copyright owners and it is a condition of accessing these publications that users recognise and abide by the legal requirements associated with these rights.

Take down policy

The Research Portal is Queen's institutional repository that provides access to Queen's research output. Every effort has been made to ensure that content in the Research Portal does not infringe any person's rights, or applicable UK laws. If you discover content in the Research Portal that you believe breaches copyright or violates any law, please contact openaccess@qub.ac.uk. 


\section{Optimum Imaging Strategies for Advanced Prostate Cancer: ASCO Guideline}

Edouard J. Trabulsi, MD${ }^{1}$; R. Bryan Rumble, MSc${ }^{2}$; Hossein Jadvar, MD, $\mathrm{PhD}^{3}$; Thomas Hope, $\mathrm{MD}^{4}$; Martin Pomper, MD, PhD Baris Turkbey, MD ${ }^{6}$; Andrew B. Rosenkrantz, MD; Sadhna Verma, MD ${ }^{8}$; Daniel J. Margolis, MD ${ }^{9}$; Adam Froemming, MD $^{10}$; Aytekin Oto, MD ${ }^{11}$; Andrei Purysko, MD ${ }^{12}$; Matthew I. Milowsky, MD ${ }^{13}$; Heinz-Peter Schlemmer, MD ${ }^{14}$; Matthias Eiber, MD ${ }^{15}$; Michael J. Morris, MD ${ }^{16}$; Peter L. Choyke, MD ${ }^{6}$; Anwar Padhani, MD ${ }^{17}$; Jorge Oldan, MD ${ }^{13}$; Stefano Fanti, MD ${ }^{18}$; Suneil Jain, NMD ${ }^{19}$; Peter A. Pinto, MD; ; Kirk A. Keegan, MD ${ }^{20}$; Christopher R. Porter, MD ${ }^{21}$; Jonathan A. Coleman, MD ${ }^{16}$; Glenn S. Bauman, MD ${ }^{22}$; Ashesh B. Jani, MD ${ }^{23}$; Jeffrey M. Kamradt, MD ${ }^{24}$; Westley Sholes, MPA; and H. Alberto Vargas, MD ${ }^{16}$

ASSOCIATED CONTENT

Appendix

Author affiliations

and support

information (if

applicable) appear

at the end of this

article.

Accepted on October

25, 2019 and

published at

ascopubs.org/journal/

jco on January 15 ,

2020: DOI https://doi.

org/10.1200/JCO.19.

02757

With panel

representation from

the American College

of Radiology,

American Urological

Association, Society

of Abdominal

Radiology, Society of

Nuclear Medicine

and Molecular

Imaging, and the

Society of Urologic

Oncology.

At the time of

publication, this

ASCO Guideline has

been endorsed by the

American College of

Radiology.

PURPOSE Provide evidence- and expert-based recommendations for optimal use of imaging in advanced prostate cancer. Due to increases in research and utilization of novel imaging for advanced prostate cancer, this guideline is intended to outline techniques available and provide recommendations on appropriate use of imaging for specified patient subgroups.

METHODS An Expert Panel was convened with members from ASCO and the Society of Abdominal Radiology, American College of Radiology, Society of Nuclear Medicine and Molecular Imaging, American Urological Association, American Society for Radiation Oncology, and Society of Urologic Oncology to conduct a systematic review of the literature and develop an evidence-based guideline on the optimal use of imaging for advanced prostate cancer. Representative index cases of various prostate cancer disease states are presented, including suspected high-risk disease, newly diagnosed treatment-naïve metastatic disease, suspected recurrent disease after local treatment, and progressive disease while undergoing systemic treatment. A systematic review of the literature from 2013 to August 2018 identified fully published English-language systematic reviews with or without meta-analyses, reports of rigorously conducted phase III randomized controlled trials that compared $\geq 2$ imaging modalities, and noncomparative studies that reported on the efficacy of a single imaging modality.

RESULTS A total of 35 studies met inclusion criteria and form the evidence base, including 17 systematic reviews with or without meta-analysis and 18 primary research articles.

RECOMMENDATIONS One or more of these imaging modalities should be used for patients with advanced prostate cancer: conventional imaging (defined as computed tomography [CT], bone scan, and/or prostate magnetic resonance imaging [MRI]) and/or next-generation imaging (NGI), positron emission tomography [PET], PET/CT, PET/MRI, or whole-body MRI) according to the clinical scenario.

J Clin Oncol 38. @ 2020 by American Society of Clinical Oncology

\section{INTRODUCTION}

The purpose of this clinical practice guideline is to provide referring and imaging clinicians (including medical oncologists, radiation oncologists, urologists, radiologists, nuclear medicine physicians, and molecular imagers), other health care practitioners, patients, and caregivers with recommendations and future directions regarding optimum imaging for patients with advanced prostate cancer based on the best available evidence. The fluid and rapidly evolving nature of the topic is acknowledged, and although regulatory approvals of some of the techniques presented are currently limited, this guideline is intended to preemptively address the ongoing barrage of studies that will most certainly transform the landscape for the management of patients with advanced prostate cancer. The term advanced prostate cancer encompasses a wide swath of patients with different disease states and clinicopathologic factors, including men with localized prostate cancer at initial diagnosis with a high or very high risk of metastasis (as defined recently in an American Urological Association/American Society of Radiation Oncology/Society of Urologic Oncology guideline ${ }^{1}$ ); men who have been treated and subsequently present with clinical, biochemical, or radiographic evidence of disease progression; and men with known metastatic disease either at initial presentation or after one or more lines of treatment. This guideline examines the optimal use of imaging for men in each of these disease states. 


\section{THE BOTTOM LINE}

\section{Optimum Imaging Strategies for Advanced Prostate Cancer: ASCO Guideline}

Endorsed by the Society of Abdominal Radiology, American College of Radiology, Society of Nuclear Medicine and Molecular Imaging, American Urological Association, American Society for Radiation Oncology, and the Society of Urologic Oncology.

\section{Guideline Question}

What are the optimum imaging options that should be offered to patients with advanced prostate cancer?

\section{Target Population}

Men with advanced prostate cancer, including newly diagnosed clinical high-risk disease, suspected or confirmed metastatic disease, recurrent disease, or progressive disease while under treatment.

\section{Target Audience}

Medical oncologists, radiation oncologists, urologists, radiologists, nuclear medicine and molecular imaging physicians, other health care practitioners such as nurses and social workers, patients, and caregivers.

\section{Methods}

An Expert Panel was convened to develop clinical practice guideline recommendations based on a systematic review of the medical literature.

\section{Definitions}

- Conventional imaging: computed tomography (CT), bone scan, and/or prostate magnetic resonance imaging (MRI).

- Next-generation imaging (NGI): positron emission tomography (PET), PET/CT, PET/MRI, whole-body MRI.

- Advanced prostate cancer: disease states/clinical scenarios described.

- Biochemical recurrence: detectable prostate-specific antigen (PSA) with a subsequent rise after radical prostatectomy or a rise of 2 above nadir PSA achieved after radiotherapy (Phoenix criteria) ${ }^{15}$; high risk without evidence of disease locally or distantly on conventional imaging where the definition of undetectable PSA is dependent on the assay used and may change over time as more-sensitive assays become available; in general, a PSA value $<0.2 \mathrm{ng} / \mathrm{mL}$ has been considered undetectable, ${ }^{1}$ while lower values (PSA $\leq 0.01 \mathrm{ng} / \mathrm{mL}$ ) have been advocated when clinically available. ${ }^{16}$

\section{Recommendations}

Recommendation 1. Imaging is recommended for all patients with advanced prostate cancer. See the recommendation under clinical question 4 for specific details according to clinical scenario (Type: evidence based, benefits outweigh harms; Evidence quality: moderate; Strength of recommendation: strong).

Recommendation 2. One or more of the following imaging modalities should be used for patients with advanced prostate cancer: conventional imaging (defined as CT, bone scan, and/or prostate MRI) and/or NGI (PET, PET/CT, PET/MRI, whole-body MRI), according to clinical scenario (Type: evidence based, benefits outweigh harms; Evidence quality: moderate; Strength of recommendation: strong).

Recommendation 3. It is recommended when choosing an imaging modality that disease states and clinical scenarios as outlined are taken into consideration, as the imaging modality may guide treatment or change clinical treatment decisions (Type: evidence based, benefits outweigh harms; Evidence quality: moderate; Strength of recommendation: strong).

\section{Newly Diagnosed Clinically High-Risk/Nery High-Risk Localized Prostate Cancer}

Recommendation 4.1. Conventional imaging negative. When conventional imaging (defined as CT, bone scan, and/or prostate MRI) is negative in patients with a high risk of metastatic disease, NGI (defined as PET, PET/CT, PET/MRI, whole-body MRI) may add clinical benefit, although prospective data are limited (Type: informal consensus, benefits/ harm ratio uncertain; Evidence quality: weak; Strength of recommendation: moderate).

Recommendation 4.2. Conventional imaging suspicious/equivocal. When conventional imaging is suspicious or equivocal, $\mathrm{NGI}$ may be offered to patients for clarification of equivocal findings or detection of additional sites of disease, which could potentially alter management, although prospective data are limited (Type: informal consensus, benefits/harm ratio uncertain; Evidence quality: weak; Strength of recommendation: moderate).

(continued on following page) 


\section{THE BOTTOM LINE (CONTINUED)}

\section{Rising PSA After Prostatectomy and Negative Conventional Imaging (either initial PSA undetectable with subsequent rise or PSA never nadirs to undetectable)}

Recommendation 4.3. Both disease states are indicative of potentially undetected, residual local, locoregional, or micrometastatic disease, and imaging options are not distinct or different between these scenarios. The goal of therapy and the potential use of salvage local therapies in these scenarios should guide the choice of imaging. For men who are not candidates or are unwilling to receive salvage local or regional therapy, additional NGI should not be offered (Type: informal consensus, benefits/harms ratio uncertain; Evidence quality: low; Strength of recommendation: moderate).

Recommendation 4.4. For men for whom salvage radiotherapy is contemplated, NGI should be offered (PSMA imaging [where available]; ${ }^{11} \mathrm{C}$-choline or ${ }^{18} \mathrm{~F}$-fluciclovine PET/CT; or PET/MRI, whole-body MRI, and/or ${ }^{18} \mathrm{~F}-\mathrm{NaF}$ PET/CT) as they have superior disease detection performance characteristics and may alter patient management (Type: evidence based, benefits outweigh harms; Evidence quality: high; Strength of recommendation: strong).

\section{Rising PSA After Radiotherapy and Negative Conventional Imaging}

Recommendation 4.5. For men in whom salvage local or regional therapy is not planned or is inappropriate, there is little evidence that NGI will alter treatment or prognosis. The role of NGI in this scenario is unclear and should not be offered, except in the context of an institutional review board-approved clinical trial (Type: informal consensus, benefits/harms ratio uncertain; Evidence quality: intermediate; Strength of recommendation: moderate).

Recommendation 4.6. For men for whom salvage local or regional therapy (eg, salvage prostatectomy, salvage ablative therapy, or salvage lymphadenectomy) is contemplated, there is evidence supporting NGI for detection of local and/or distant sites of disease. Findings on NGI could guide management in this setting (eg, salvage local, systemic or targeted treatment of metastatic disease, combined local and metastatic therapy). PSMA imaging (where available), ${ }^{11} \mathrm{C}-\mathrm{choline}$ or ${ }^{18} \mathrm{~F}$-fluciclovine PET/CT or PET/MRI, whole-body MRI, and/or ${ }^{18} \mathrm{~F}-\mathrm{NaF}$ PET/CT have superior disease detection performance characteristics compared with conventional imaging and alter patient management, although data are limited (Type: evidence based, benefits outweigh harms; Evidence quality: intermediate; Strength of recommendation: moderate).

\section{Metastatic Prostate Cancer at Initial Diagnosis or After Initial Treatment, Hormone Sensitive}

Recommendation 4.7. In the initial evaluation of men presenting with hormone-sensitive disease with demonstrable metastatic disease on conventional imaging, there is a potential role for NGI to clarify the burden of disease and potentially shift the treatment intent from multimodality management of oligometastatic disease to systemic anticancer therapy alone or in combination with targeted therapy for palliative purposes, but prospective data are limited (Type: informal consensus, benefits/harms ratio uncertain; Evidence quality: intermediate; Strength of recommendation: moderate).

\section{Nonmetastatic Castration-Resistant Prostate Cancer}

Recommendation 4.8. For men with nonmetastatic castration-resistant prostate cancer (CRPC), NGI can be offered only if a change in the clinical care is contemplated. Assuming patients have received or are ineligible for local salvage treatment options, NGI may clarify the presence or absence of metastatic disease, but the data on detection capabilities of $\mathrm{NGl}$ in this setting and impact on management are limited (Type: consensus, benefits/harms ratio uncertain; Evidence quality: weak; Strength of recommendation: moderate).

\section{Metastatic CRPC}

Recommendation 4.9. PSA progression. As recommended by the Prostate Cancer Working Group 3 consensus statements, PSA progression alone for men on treatment of metastatic CRPC should not be the sole reason to change therapy. Conventional imaging can be used for initial evaluation of PSA progression and should be continued to facilitate changes/comparisons and serially to assess for development of radiographic progression (Type: informal consensus, benefits/harms ratio uncertain; Evidence quality: intermediate; Strength of recommendation: strong).

Recommendation 4.10. The use of NGI in this cohort is unclear, with a paucity of prospective data. When a change in clinical care is contemplated, in an individualized manner, and there is a high clinical suspicion of subclinical metastasis despite negative conventional imaging, the use of NGI could be contemplated, especially in the setting of a clinical trial (Type: informal consensus, benefits/harms ratio uncertain; Evidence quality: insufficient; Strength of recommendation: weak).

$$
\text { (continued on following page) }
$$




\section{THE BOTTOM LINE (CONTINUED)}

Recommendation 4.11. Radiographic progression on conventional imaging. In men with metastatic CRPC with clear evidence of radiographic progression on conventional imaging while on systemic therapy, NGI should not be routinely offered. NGI may play a role if performed at baseline to facilitate comparison of imaging findings/extent of progression of disease (Type: consensus, benefits/harms ratio uncertain; Evidence quality: insufficient; Strength of recommendation: moderate).

\section{Additional Resources}

More information, including a Data Supplement with additional evidence tables, slide sets, and clinical tools and resources, is available at www.asco.org/genitourinary-cancer-guidelines. The Methodology Manual (available at www.asco.org/guidelinemethodology) provides additional information about the methods used to develop this guideline. Patient information is available at www.cancer.net.

ASCO believes that cancer clinical trials are vital to inform medical decisions and improve cancer care, and that all patients should have the opportunity to participate.

Prostate cancer is the most common nondermatologic cancer in men. In 2019, it was estimated that there would be 174,650 new cases in the United States, and in spite of advances in diagnosis and treatment, an estimated 31,620 deaths would occur. ${ }^{2}$ In addition to its prevalence, prostate cancer poses unique challenges, including a distinct clinical disease state characterized by an elevated serum prostate-specific antigen (PSA) consistent with recurrent disease without findings of metastases on historically conventional imaging studies and difficulty in monitoring patients with metastatic bone disease due to the poor test characteristics of conventional bone imaging. For these reasons, coupled with increasing evidence for local salvage therapy or metastasis-directed therapy or increasingly effective systemic therapies that have been shown to be beneficial ever earlier in the natural history of the disease, there is great interest in identifying better imaging strategies to inform the optimum management for patients with advanced prostate cancer.

The predilection for prostate cancer to metastasize to bone and lymph nodes requires both bone and soft tissue imaging techniques to assess for staging and to monitor for response to therapy and progression of disease. Conventional standard imaging modalities include

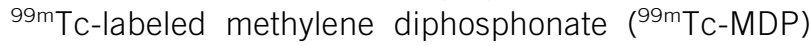
bone scan and computerized tomography (CT) or magnetic resonance imaging (MRI). The relatively poor specificity of ${ }^{99 \mathrm{~m} T \mathrm{~T}-M D P}$ relates to the fact that MDP adsorbs onto the crystalline hydroxyapatite mineral of bone and is not prostate cancer-specific, thus providing only an indirect measure of tumor activity based on osteoblastic activity in the tumor microenvironment. In addition, the interpretation of a bone scan is subjective, relying on manual assessments of lesion number, size, and intensity. Such subjective assessments are problematic in the setting of clinical trials and lead to difficulty in accurately interpreting treatment effects. Patients treated with effective therapy can have paradoxically worsening scans in the face of response to treatment. ${ }^{3}$ Standard criteria have been developed to standardize bone scan interpretation and to distinguish response from progression. ${ }^{4,5}$ These semiquantitative criteria have been shown to be feasible, have demonstrated in prospective studies to correlate with survival, and have been recognized as clinically relevant to warrant their use by regulatory authorities for drug approval. ${ }^{6-8}$ In addition, quantitative tools for the assessment of bone scan data have been developed, including the automated bone scan index (BSI), which represents the total tumor burden as the fraction of the total skeleton weight. ${ }^{9-11}$ These quantitative tools improve bone scan interpretation but are still subject to the relatively poor specificity of

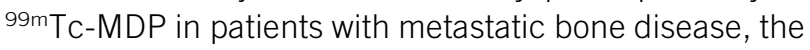
inability to detect nodal and visceral metastatic disease, and the poor ability of ${ }^{99 \mathrm{~m} T c-M D P}$ and CT or MRI to detect metastatic disease in patients with early biochemical recurrence.

In the same way that molecularly targeted therapies have transformed decision making of many diseases, advances in nuclear medicine and molecular imaging are poised to re-invent the way in which we diagnose, stage, and monitor response to therapy in patients with prostate cancer. These next-generation imaging (NGI) modalities promise improved diagnostic accuracy for staging prostate cancer, especially at lower tumor burdens. New radiopharmaceuticals coupled to prostate cancer-specific targets, such as prostate-specific membrane antigen (PSMA), are defined in this guideline as external domain PSMA-binding ligands labeled with the positron emission tomography (PET)-emitters ${ }^{68} \mathrm{Ga}$ and ${ }^{18} \mathrm{~F}$, exclusive of the PSMA antibody capromab pendetide that binds the internal domain of PSMA. These have demonstrated improvements in the sensitivity for the detection of metastatic disease and the monitoring of a treatment effect in patients with advanced 
prostate cancer compared with conventional imaging. The recent US Food and Drug Administration (FDA) approvals of ${ }^{11} \mathrm{C}$-choline and ${ }^{18} \mathrm{~F}$-fluciclovine for PET imaging in men with a suspected prostate cancer recurrence based on an elevated PSA following prior treatment represent watershed moments in the development of novel imaging tools for advanced prostate cancer. ${ }^{12}$

While patients and clinicians may find additional imaging, especially NGI, attractive for advanced prostate cancer in a variety of clinical states, there are several disadvantages associated with their use. These NGI modalities are costly and are not routinely covered by all third-party payers in the United States. Their routine use could significantly increase overall expenditure for prostate cancer care. While the NGI modalities have excellent sensitivity to detect low-burden disease, false-positive results may lead to incorrect patient management in some patients. Moreover, the presence of $\mathrm{NGl}$-detected lesions may trigger additional procedures, imaging modalities, and invasive biopsies, which carry risks and additional cost in addition to mental burden and uncertainty for patients. Figure 1 provides the imaging algorithm for high/very highrisk disease at initial presentation (per National Comprehensive Cancer Network). Figure 2 provides the imaging algorithm for patients with rising PSA after local treatment.

Another important consideration is concern for the Will Rogers phenomenon, which may ensue when patients who are classified as nonmetastatic with conventional imaging become reclassified as metastatic after NGI. This may alter treatment decisions with unknown consequences on the overall disease course. ${ }^{13}$ The Will Rogers phenomenon also makes comparison with historical standards and controls very difficult, with the risk of artifactual changes in stage-specific survival. ${ }^{14}$

This guideline addresses the goals of imaging in advanced prostate cancer, considering conventional imaging techniques and newer NGI modalities, as well as unmet needs, the potential impact of imaging according to different advanced prostate cancer clinical disease states, and the type of imaging that is most appropriate in each scenario. The guideline focuses on appropriate utility of imaging for advanced prostate cancer and not on treatment decisions; any discussion of treatment decisions are given in the context of the impact of imaging on clinical decision making. Evaluation of treatment options and treatment decisions are beyond the scope of this guideline.

\section{GUIDELINE QUESTIONS}

This clinical practice guideline addresses four overarching clinical questions:

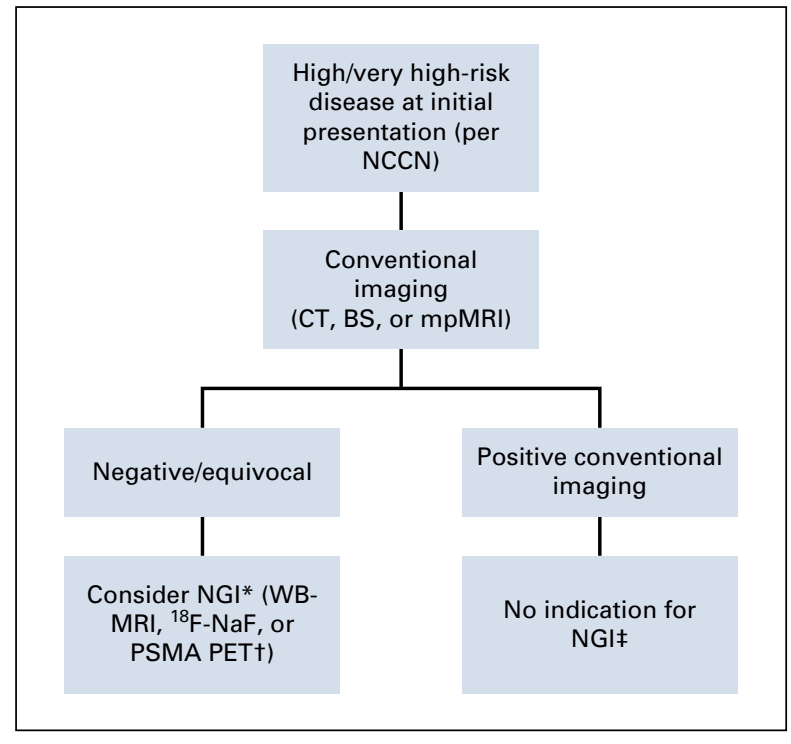

FIG 1. Imaging algorithm for high/very high-risk disease at initial presentation (per National Comprehensive Cancer Network [NCCN]). (*) Suspicious findings on NGI would influence treatment decisions in patients with advanced prostate cancer and negative conventional imaging, opening the scope for multimodality treatment of primary and oligometastatic disease or systemic therapy for more extensive metastatic states, although prospective data are limited. $(\dagger)$ There is enthusiasm for the potential added value of PSMA PET/CT and PET/MRI for the assessment of the local and metastatic extent of prostate cancer in this context, although PSMA imaging is not currently FDA approved and should thus be only performed as part of a clinical trial or other controlled research setting. ( $\ddagger) \mathrm{NGl}$ could offer clinical benefit in this scenario by redefining the true extent of disease and shifting treatment decisions accordingly, although prospective data in this context are limited. BS, bone scintigraphy; CT, computed tomography; mpMRI, multiparametric magnetic resonance imaging; NGI, next-generation imaging; PET, positron emission tomography; PSMA, prostatespecific membrane antigen; WB, whole body.

1. What is the goal of imaging in advanced prostate cancer?

2. What imaging techniques are available for imaging advanced prostate cancer?

3. What are the unmet needs and potential impact of imaging according to different advanced prostate cancer disease states?

4. When and what type of imaging is appropriate in each scenario?

The recommendations are framed according to these clinical scenarios:

A. Newly diagnosed clinically high-risk/very high-risk prostate cancer

i. Conventional imaging negative

ii. Conventional imaging suspicious/equivocal

B. Rising PSA after prostatectomy and negative conventional imaging

i. Initial PSA undetectable with subsequent rise

ii. PSA never nadirs to undetectable 


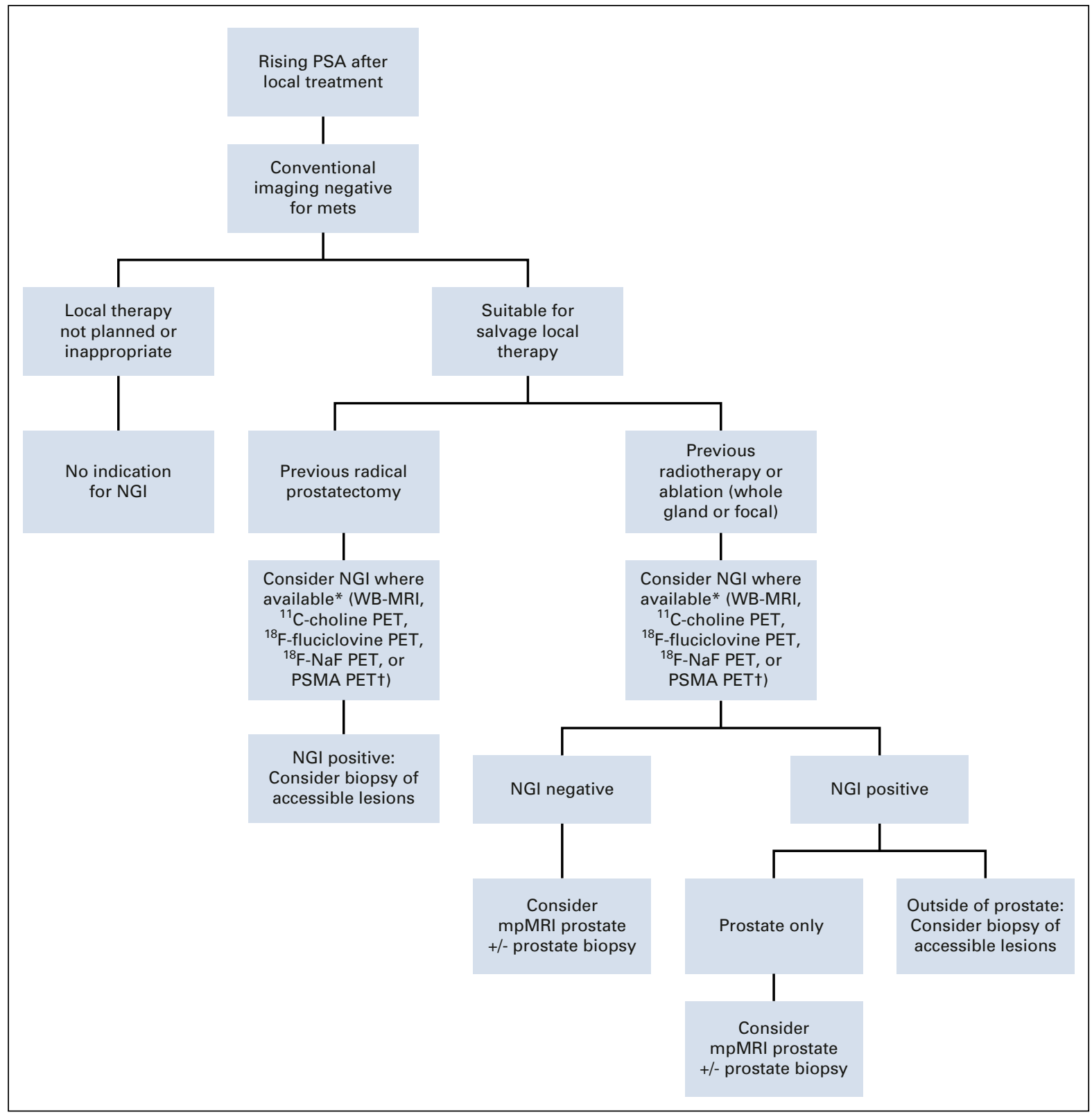

FIG 2. Imaging algorithm for patients with rising prostate-specific antigen (PSA) after local treatment. (*) For men for whom salvage local therapy (e.g. salvage radiation, salvage prostatectomy) is an option, there is evidence supporting the use of NGI to assess local or distant sites of disease, which may guide therapy away from salvage local therapy if indicative of distant metastatic disease. ( $\dagger$ ) There is enthusiasm for the potential added value of PSMA PET/CT and PET/MRI for the assessment of the local and metastatic extent of prostate cancer in this context, although PSMA imaging is not currently FDA approved and should thus be only performed as part of a clinical trial or other controlled research setting. Mets, metastatic disease; mpMRI, multiparametric magnetic resonance imaging; NGI, next-generation imaging; PET, positron emission tomography; PSMA, prostate-specific membrane antigen; WB, whole body.

C. Rising PSA after radiotherapy and negative conventional imaging

D. Metastatic prostate cancer at initial diagnosis or after treatment, hormone sensitive

E. Nonmetastatic castration-resistant prostate cancer (CRPC)

F. Metastatic CRPC
i. PSA progression
ii. Radiographic progression on conventional imaging

\section{METHODS}

\section{Guideline Development Process}

This systematic review-based guideline product was developed by a multidisciplinary Expert Panel comprising members from all the partner organizations (herein, the Expert Panel), which included a patient representative and an ASCO guidelines staff member with health research methodology expertise (Appendix Table A1, online only). 
The Expert Panel met in person several times as well as via teleconference and/or webinar and corresponded through e-mail. Based upon the consideration of the evidence, the authors were asked to contribute to the development of the guideline, provide critical review, and finalize the guideline recommendations. The guideline recommendations were sent for an open comment period of 2 weeks, allowing the public to review and comment on the recommendations after submitting a confidentiality agreement. These comments were taken into consideration while finalizing the recommendations. Members of the Expert Panel were responsible for reviewing and approving the penultimate version of guideline, which was then circulated for external review and submitted to Journal of Clinical Oncology (JCO) for editorial review and consideration for publication. All ASCO guidelines are ultimately reviewed and approved by the Expert Panel and the ASCO Clinical Practice Guideline Committee (CPGC) prior to publication. All funding for the administration of the project was provided by ASCO.

The recommendations were developed by using a systematic review (2013 through September 2017 and then updated in August 2018) of systematic reviews with or without meta-analysis, phase III randomized clinical trials (RCTs), comparative nonrandomized studies, and clinical experience. Articles reporting on other study designs were considered if they included outcomes, interventions, or comparisons unavailable elsewhere. Articles were selected for inclusion in the systematic review of the evidence based on the following criteria:

- Population: men with advanced prostate cancer

- Fully published or recent meeting presentations of English-language systematic reviews with or without meta-analyses, reports of rigorously conducted phase III RCTs that compared two or more imaging modalities, and noncomparative studies that report on the efficacy of a single imaging modality

- Reported on a minimum number of patients (>50)

Articles were excluded from the systematic review if they were (1) meeting abstracts not subsequently published in peer-reviewed journals; (2) editorials, commentaries, letters, news articles, case reports, or narrative reviews; or (3) published in a non-English language. The guideline recommendations are crafted, in part, using the Guidelines Into Decision Support (GLIDES) methodology and accompanying BRIDGE-Wiz software. ${ }^{17}$ In addition, a guideline implementability review is conducted. Based on the implementability review, revisions were made to the draft to clarify recommended actions for clinical practice (see Data Supplement 5: Implementability Survey Results). Ratings for the type and strength of recommendation, evidence, and potential bias are provided with each recommendation.
Detailed information about the methods used to develop this guideline is available in the Methodology Manual at www.asco.org/guideline-methodology. Other information may be found in the Data Supplement, including the clinical questions (Data Supplement 1), the search strategy (Data Supplement 2), the QUORUM diagram (Data Supplement 3), the study quality assessment (Data Supplement $4 A$ ), and the study risk of bias assessment (Data Supplement 4B).

The ASCO Expert Panel and guidelines staff will work with co-chairs to keep abreast of any substantive updates to the guideline. Based on formal review of the emerging literature, ASCO will determine the need to update. This is the most recent information as of the publication date.

\section{Guideline Disclaimer}

The Clinical Practice Guidelines and other guidance published herein are provided by the American Society of Clinical Oncology, Inc. (ASCO) to assist providers in clinical decision making. The information herein should not be relied upon as being complete or accurate, nor should it be considered as inclusive of all proper treatments or methods of care or as a statement of the standard of care. With the rapid development of scientific knowledge, new evidence may emerge between the time information is developed and when it is published or read. The information is not continually updated and may not reflect the most recent evidence. The information addresses only the topics specifically identified therein and is not applicable to other interventions, diseases, or stages of diseases. This information does not mandate any particular course of medical care. Further, the information is not intended to substitute for the independent professional judgment of the treating provider, as the information does not account for individual variation among patients. Recommendations reflect high, moderate, or low confidence that the recommendation reflects the net effect of a given course of action. The use of words like "must," "must not," "should," and "should not" indicates that a course of action is recommended or not recommended for either most or many patients, but there is latitude for the treating physician to select other courses of action in individual cases. In all cases, the selected course of action should be considered by the treating provider in the context of treating the individual patient. Use of the information is voluntary. ASCO provides this information on an "as is" basis and makes no warranty, expressed or implied, regarding the information. ASCO specifically disclaims any warranties of merchantability or fitness for a particular use or purpose. ASCO assumes no responsibility for any injury or damage to persons or property arising out of or related to any use of this information, or for any errors or omissions.

\section{Guideline and Conflicts of Interest}

The Expert Panel was assembled in accordance with ASCO's Conflict of Interest Policy Implementation 
for Clinical Practice Guidelines ("Policy," found at http://www.asco.org/rwc). All members of the Expert Panel completed ASCO's disclosure form, which requires disclosure of financial and other interests, including relationships with commercial entities that are reasonably likely to experience direct regulatory or commercial impact as a result of promulgation of the guideline. Categories for disclosure include employment; leadership; stock or other ownership; honoraria, consulting or advisory role; speaker's bureau; research funding; patents, royalties, other intellectual property; expert testimony; travel, accommodations, expenses; and other relationships. In accordance with the Policy, the majority of the members of the Expert Panel did not disclose any relationships constituting a conflict under the Policy.

\section{RESULTS}

\section{Evidence Overview}

A total of 35 articles comprising 17 systematic reviews ${ }^{18-34}$ with or without meta-analysis and 18 primary research articles $^{35-52}$ met eligibility criteria and form the evidentiary basis for the guideline recommendations. Table 1 provides a brief description of each. Other articles were brought in for discussion purposes as warranted.

\section{Systematic Reviews}

The 17 systematic reviews ${ }^{18-34}$ underwent evaluation of their pooled results from studies published between 1998 and 2017. Eight reviews ${ }^{19,23,24,26,28,30,32,33}$ compared various PET radiopharmaceuticals labeled with ${ }^{18} \mathrm{~F}$ or ${ }^{11} \mathrm{C}$; three reviews ${ }^{18,20,22}$ reported on ${ }^{68} \mathrm{Ga}-\mathrm{PSMA}$ binding ligand PET alone using various radiopharmaceuticals; two reviews ${ }^{21,34}$ compared $\mathrm{PET} / \mathrm{CT}$ using various radiopharmaceuticals with MRI; two reviews ${ }^{25,31}$ reported on ${ }^{68} \mathrm{Ga}$ PSMA binding ligand PET and MRI alone using various radiopharmaceuticals; and two ${ }^{27,29}$ reported on a pooled analysis that included ${ }^{11} \mathrm{C} \mathrm{PET} / \mathrm{CT}, \mathrm{MRI}$, bone singlephoton emission computed tomography (SPECT), and bone scintigraphy with various radiopharmaceuticals. Eleven of these reviews ${ }^{18,19,21,22,24,25,27-29,32,34}$ reported on recurrence, staging, or restaging, five ${ }^{20,23,26,30,33}$ reported on diagnostic utility, and one ${ }^{31}$ reported on the results of pooling outcomes related to determining extraprostatic extension and seminal vesicle involvement. Table 1 provides more detail.

\section{Primary Research Articles}

The 18 primary research articles ${ }^{35-52}$ obtained reported on studies published between $2014^{52}$ and $2018^{35}$ that accrued patients between 2007 and 2017 (six articles ${ }^{38,41,44,47,48,51}$ did not report on the years the study was open to accrual). Seven of these articles $37,38,44,48-50,52$ used a prospective design, and 11 articles ${ }^{35,36,39-43,45-47,51}$ were retrospective studies. Five of these articles ${ }^{35,36,38,40,43}$ reported on studies investigating PSMA binding Iigand imaged with PET ( $v$ other PSMA binding ligands imaged with PET, histology, or other end points), eight articles $37,39,41,42,45,46,49,51$ reported on PSMA binding ligand with PET imaging modalities compared with PET/CT or PET/MRI alone, and five articles $44,47,48,50,52$ reported on ${ }^{18} \mathrm{~F}$ or ${ }^{11} \mathrm{C} \mathrm{PET/CT}$. As no pooling was performed, studies that were also included in any of the previously summarized systematic reviews were retained and are reported on. Table 1 provides more detail.

\section{Study Quality Assessment}

Study design aspects related to individual study quality, strength of evidence, strength of recommendations, and risk of bias were assessed. Findings were that the evidence obtained was a representative body of literature that recommendations could be based on. For the systematic reviews, the risk of bias was lower than that of the primary articles, mostly due to the study designs used in assessments of diagnostic utility (predominantly retrospective designs with within-group comparisons). See Data Supplement 4A (Study Quality Assessment, systematic reviews with or without meta-analysis) and Data Supplement 4B (Study Risk of Bias Assessment, primary studies) for the complete results of the quality assessment.

\section{Study Results}

Systematic reviews. Seventeen systematic reviews ${ }^{18-34}$ were obtained, and the main findings (Table 2) include the following:

- In a systematic review comprising 56 studies and 7,329 patients published between 2005 and 2015, Liu et $\mathrm{al}^{23}$ found ${ }^{18} \mathrm{~F}$-fluorocholine (FCH) PET/CT superior to ${ }^{11} \mathrm{C}$-choline, ${ }^{11} \mathrm{C}$-acetate, and ${ }^{18} \mathrm{~F}$-fludeoxyglucose (FDG) for both sensitivity and specificity in the initial detection of prostate cancer.

- Treglia et al, ${ }^{28}$ in a systematic review comprising 14 studies and 1,869 patients published between 2008 and 2013 , found ${ }^{18} \mathrm{~F}$-choline or ${ }^{11} \mathrm{C}$-choline PET/CT detection rates were affected by PSA doubling times and rising PSA velocity, and these two factors should be taken into account when deciding which patients are appropriate for restaging with choline PET/CT.

- In a systematic review of 47 studies involving 3,167 patients published between 1998 and 2013, von Eyben et $\mathrm{al}^{26}$ found either ${ }^{11} \mathrm{C}$-choline PET/CT or ${ }^{18} \mathrm{~F}$-choline PET/CT informative for recurrence detection in patients with PSA levels between 1 and $50 \mathrm{ng} / \mathrm{mL}$. Performing either test also resulted in a change to the treatment plan in $41 \%$ of patients (381 of 938), while $25 \%$ (101 of 404) experienced a complete PSA response.

- Mohsen et al, ${ }^{30}$ in a systematic review of 25 studies (number of patients not reported), reported pooled sensitivity of $75.1 \%(95 \% \mathrm{Cl}, 69.8 \%$ to $79.8 \%)$ and pooled specificity of $75.8 \%(95 \% \mathrm{Cl}, 72.4 \%$ to $78.9 \%)$ in the detection of initial prostate cancer and a pooled sensitivity of $64 \%$ (95\% Cl, 59\% to 69\%) and pooled 


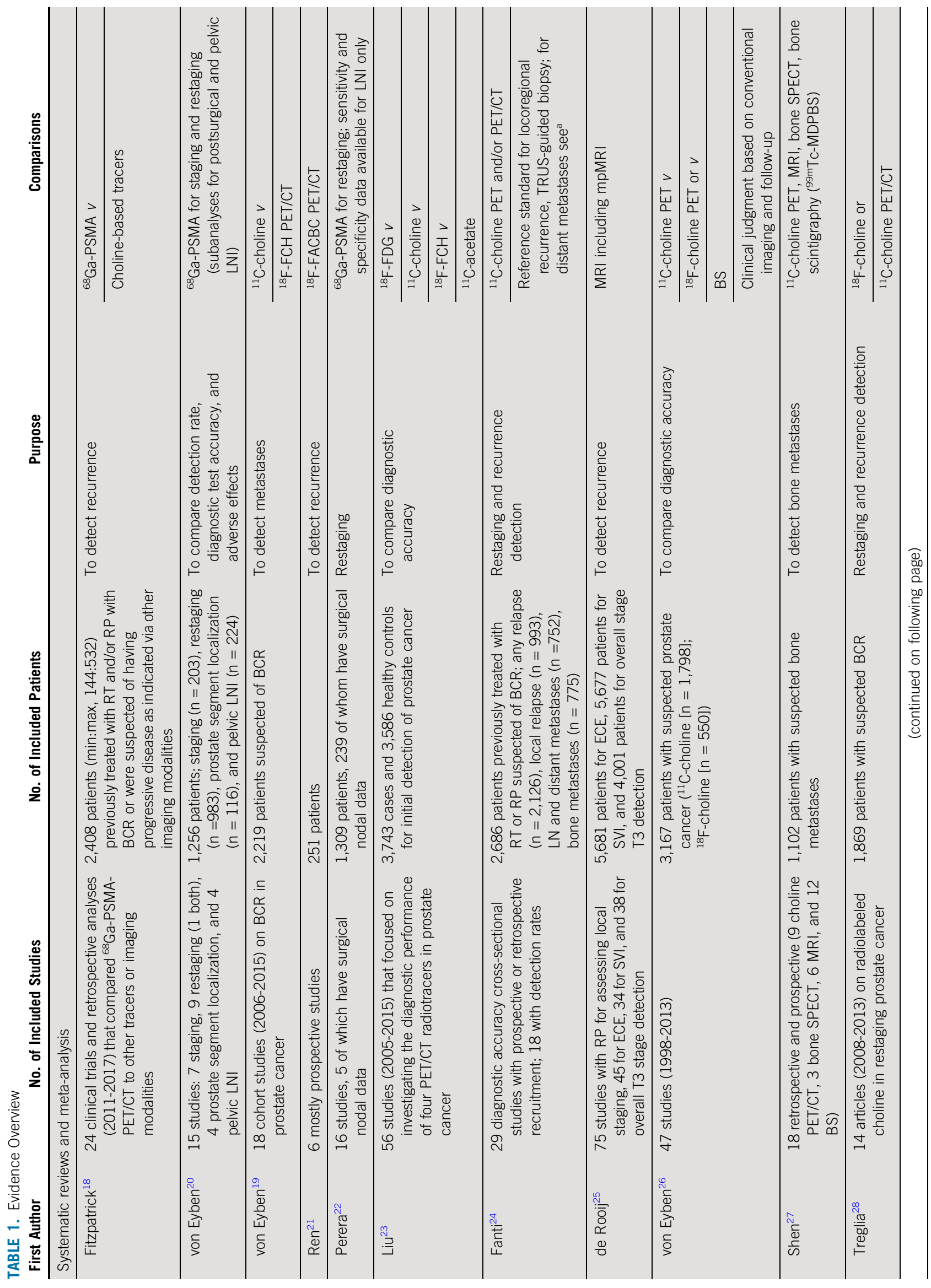




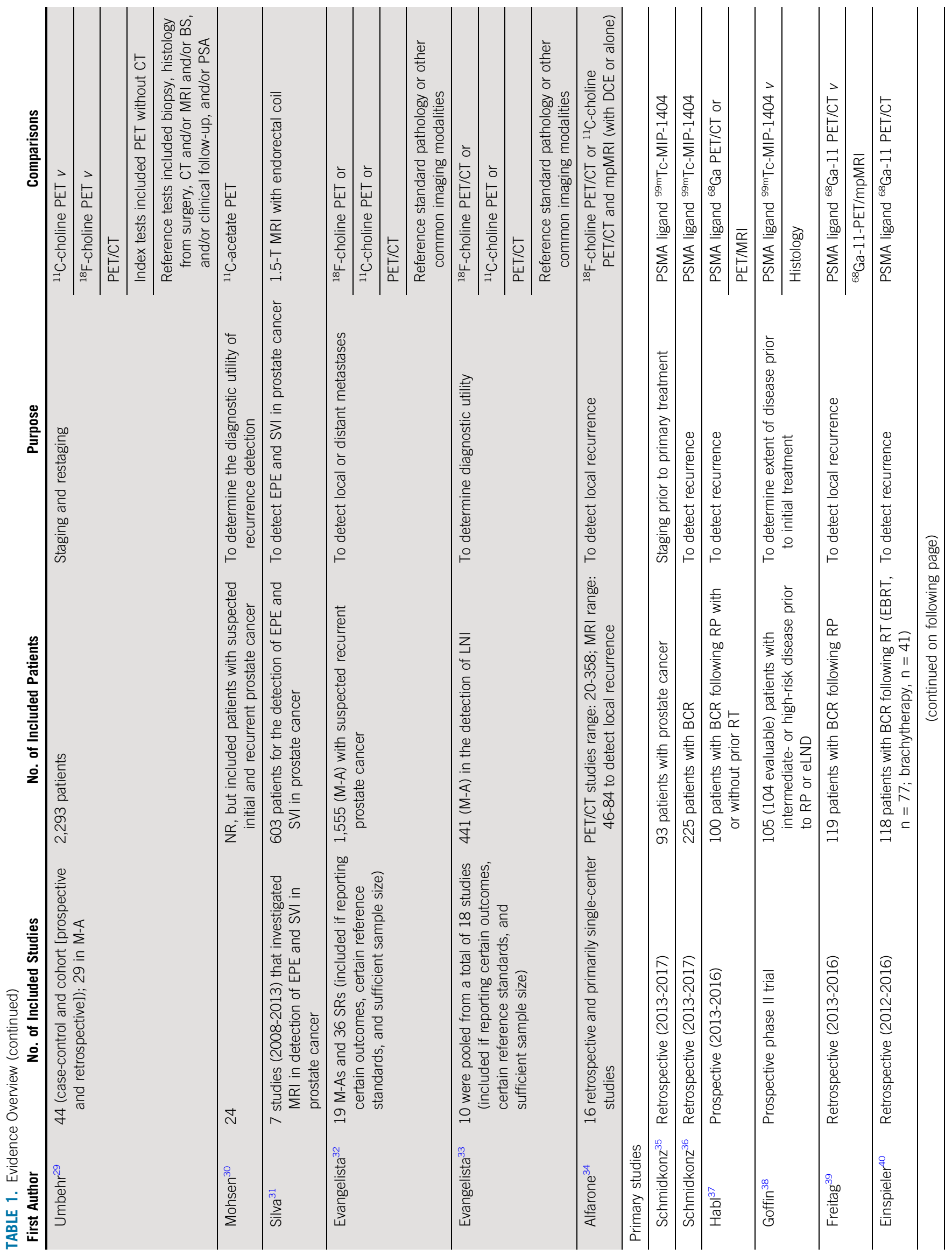




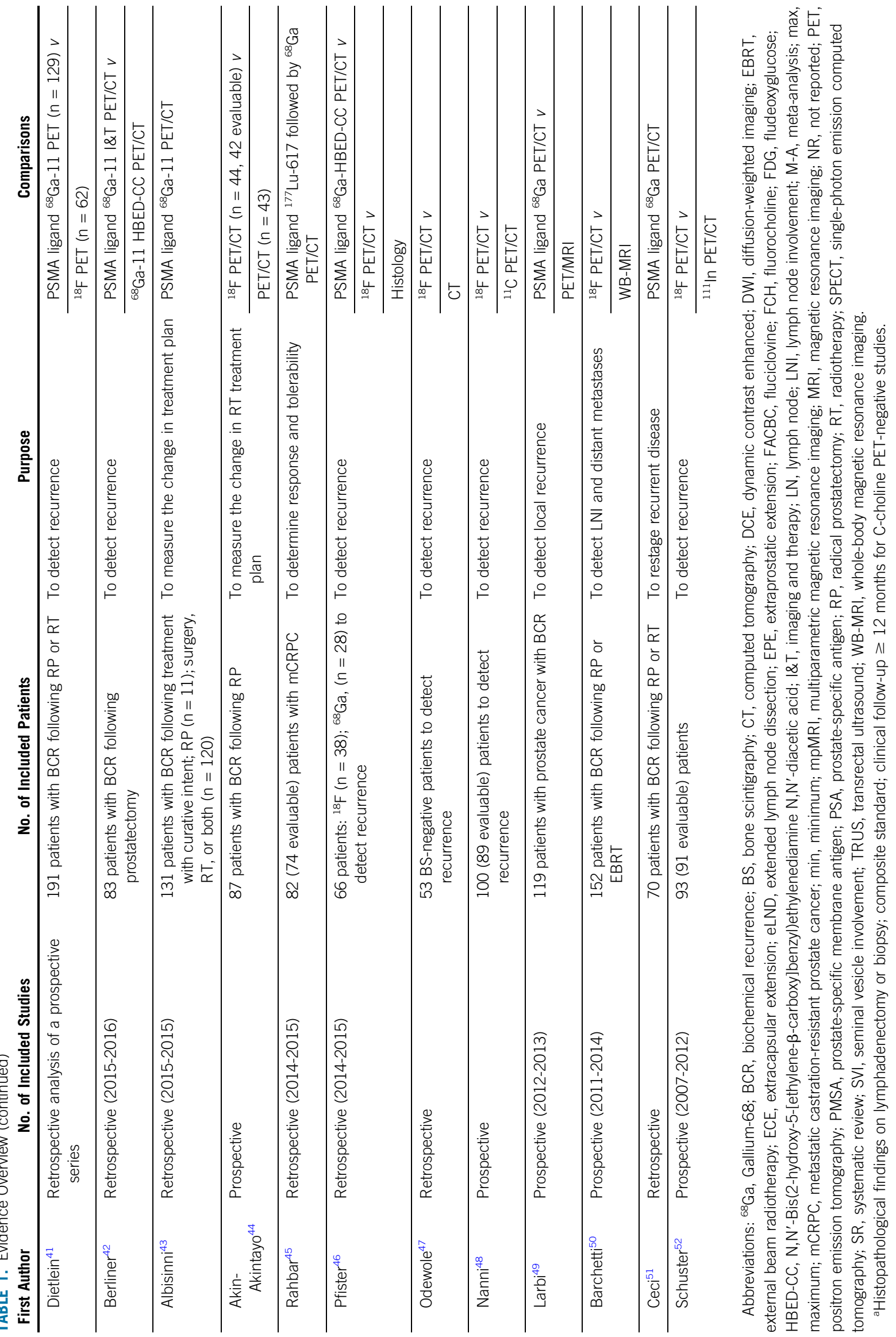




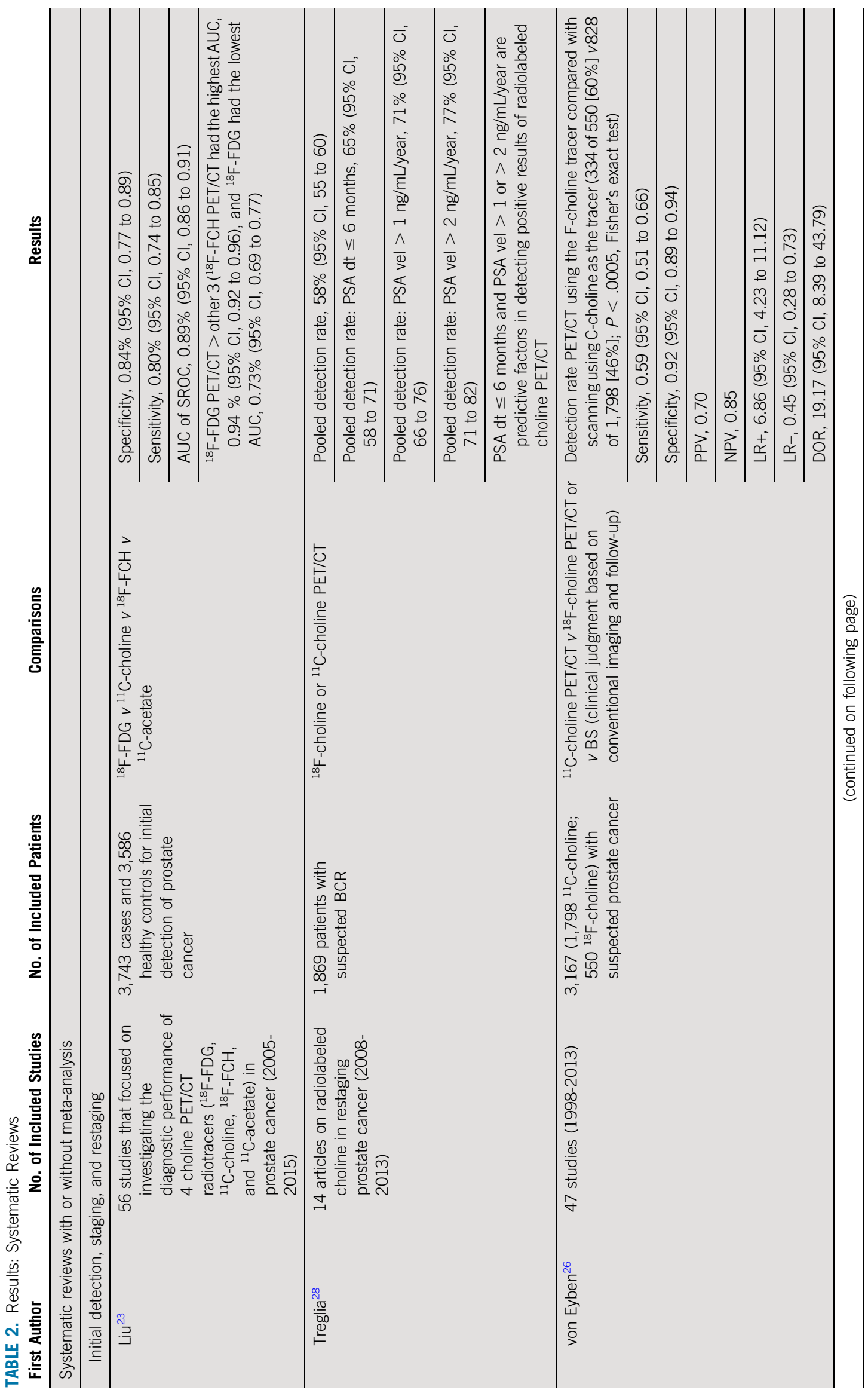




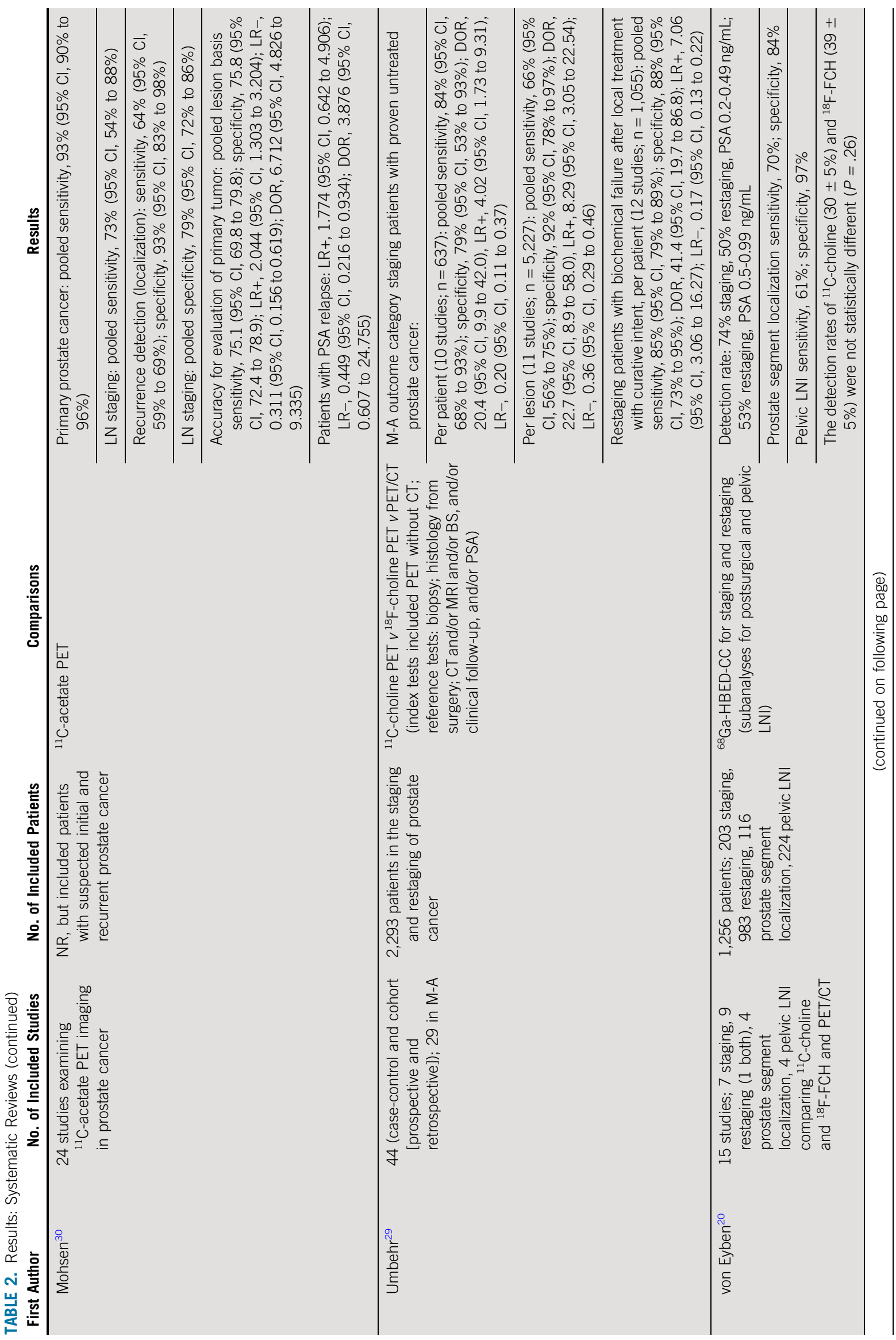




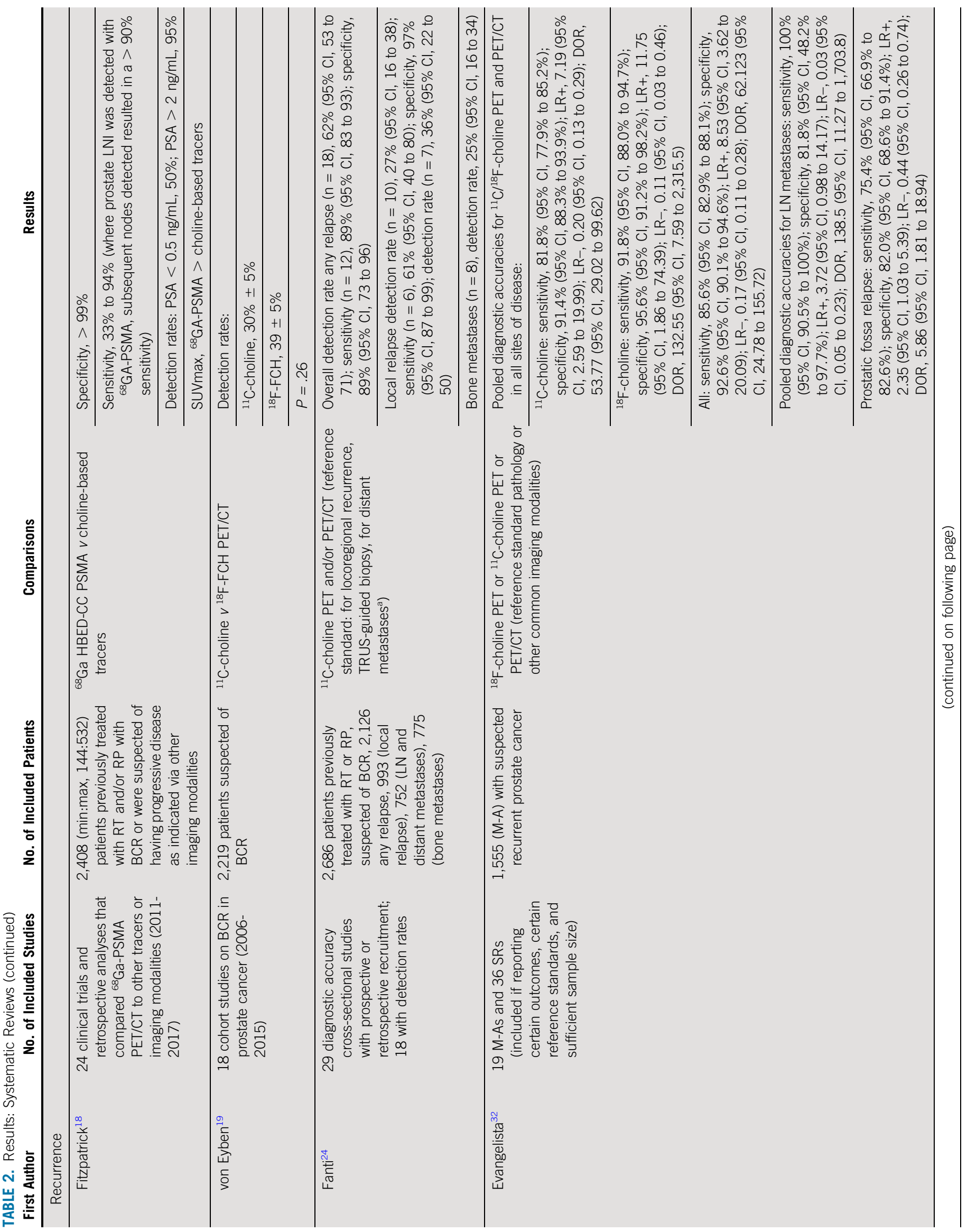




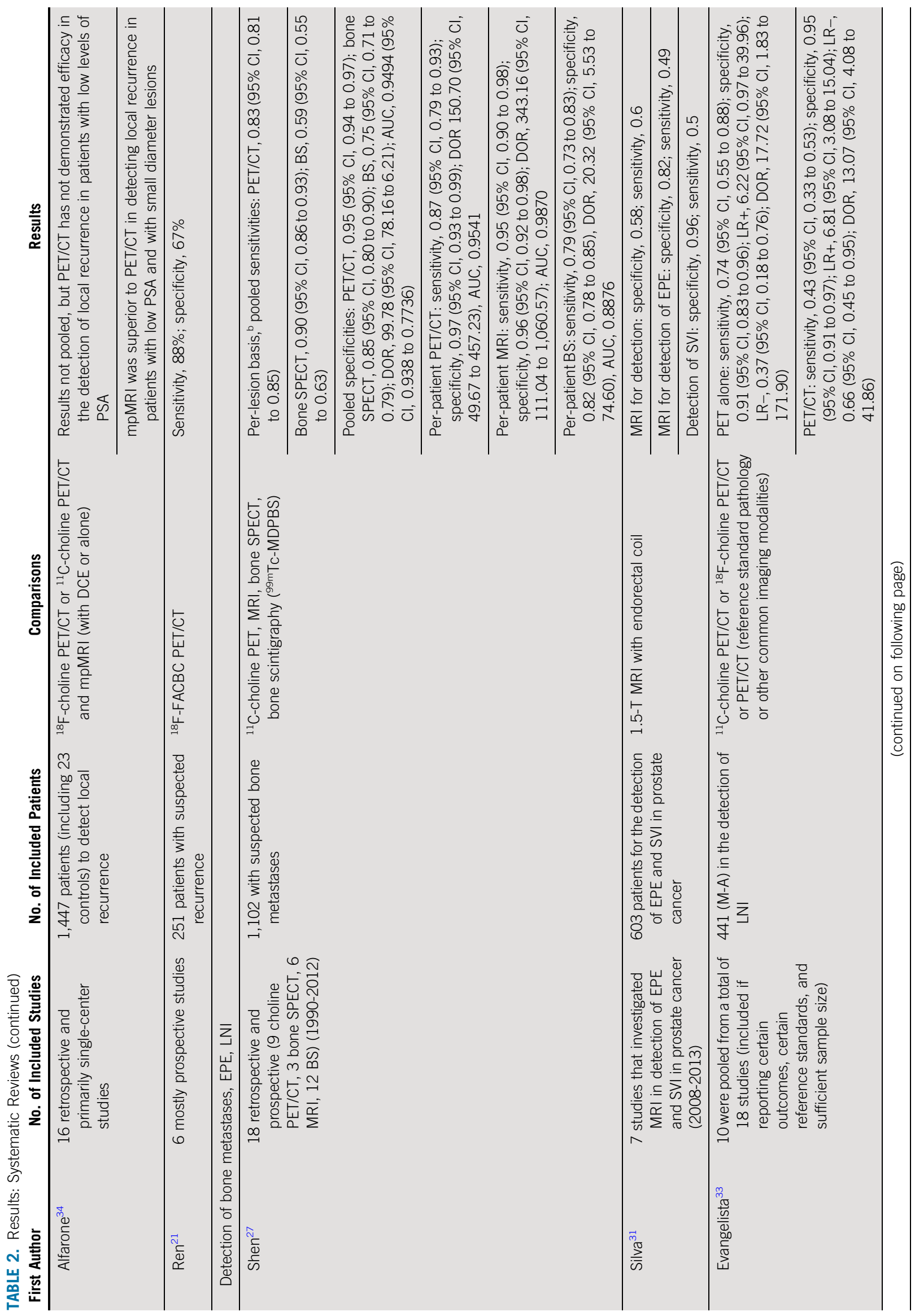




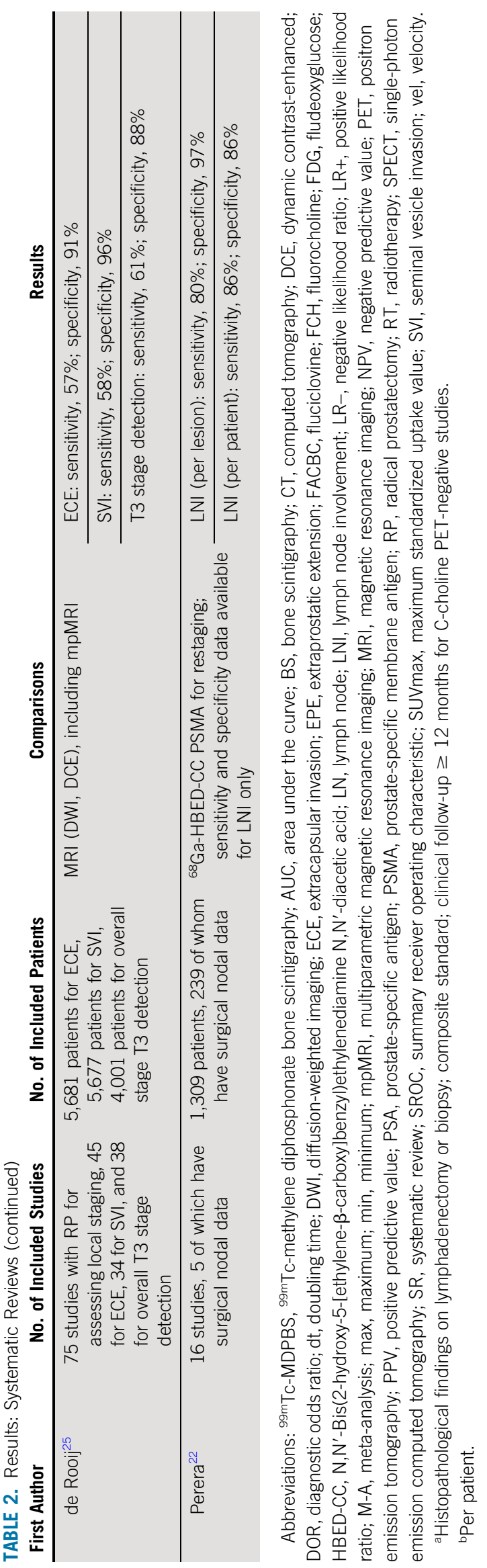

16 ○ 2020 by American Society of Clinical Oncology 


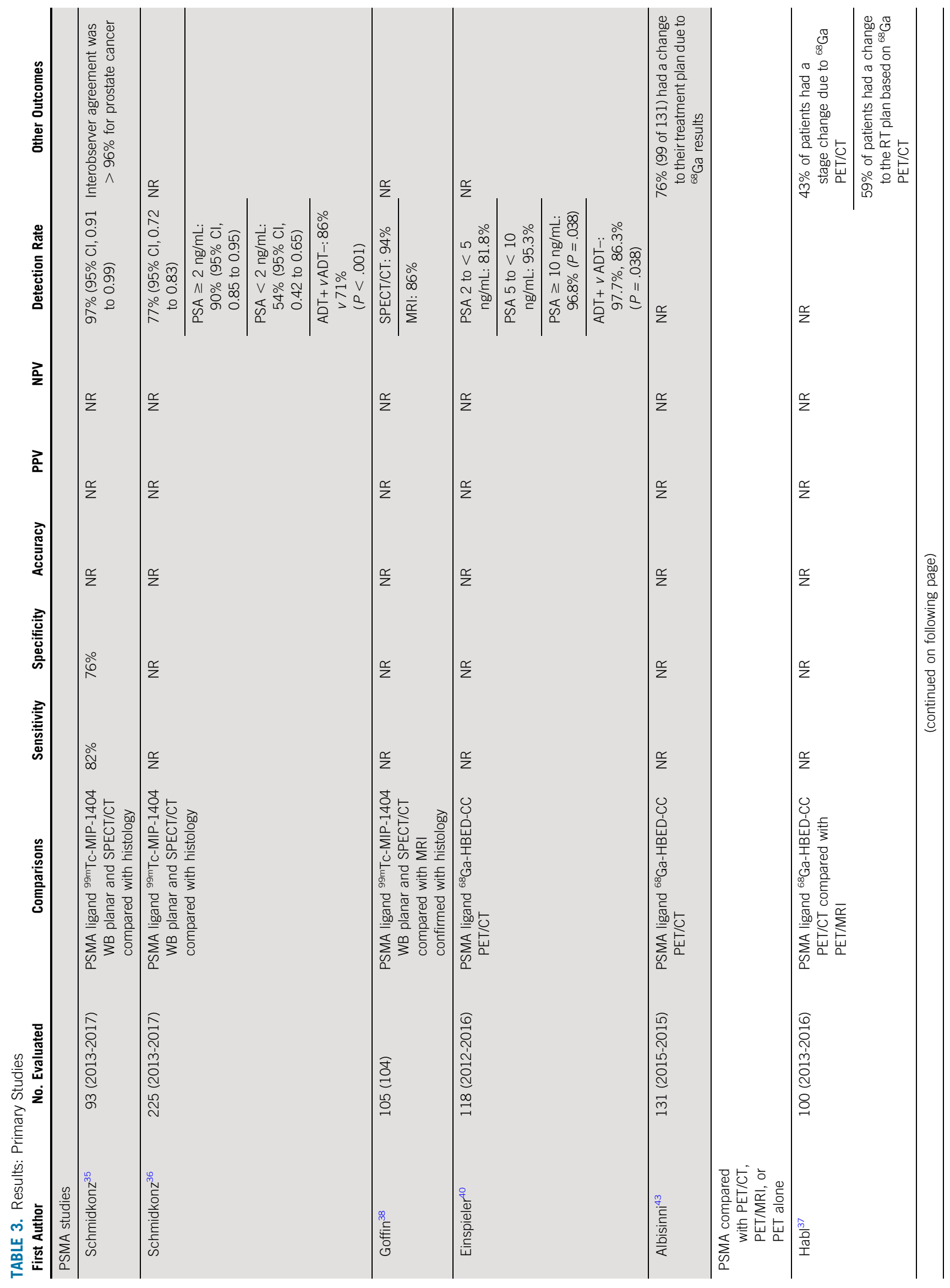




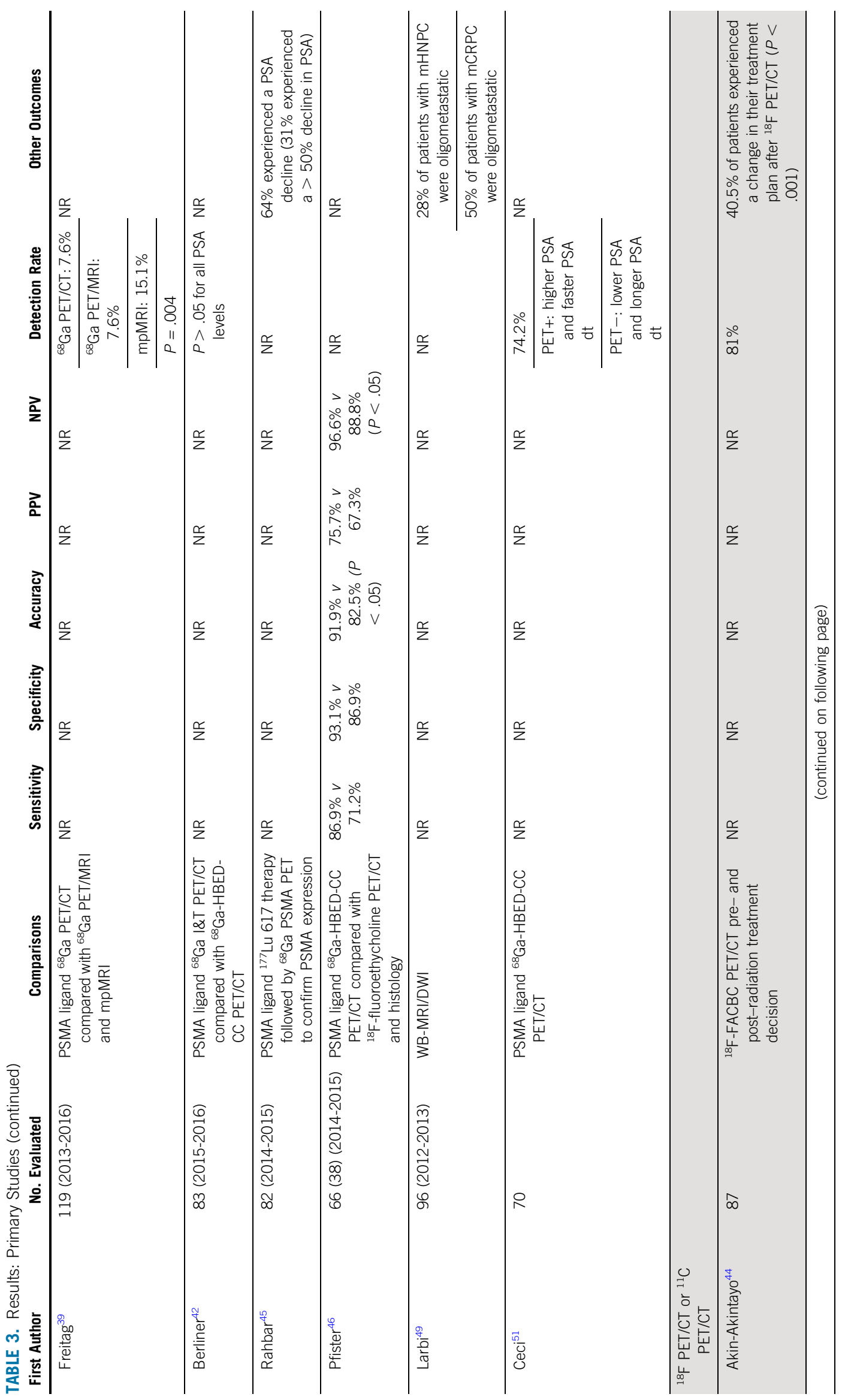

18 ○ 2020 by American Society of Clinical Oncology 


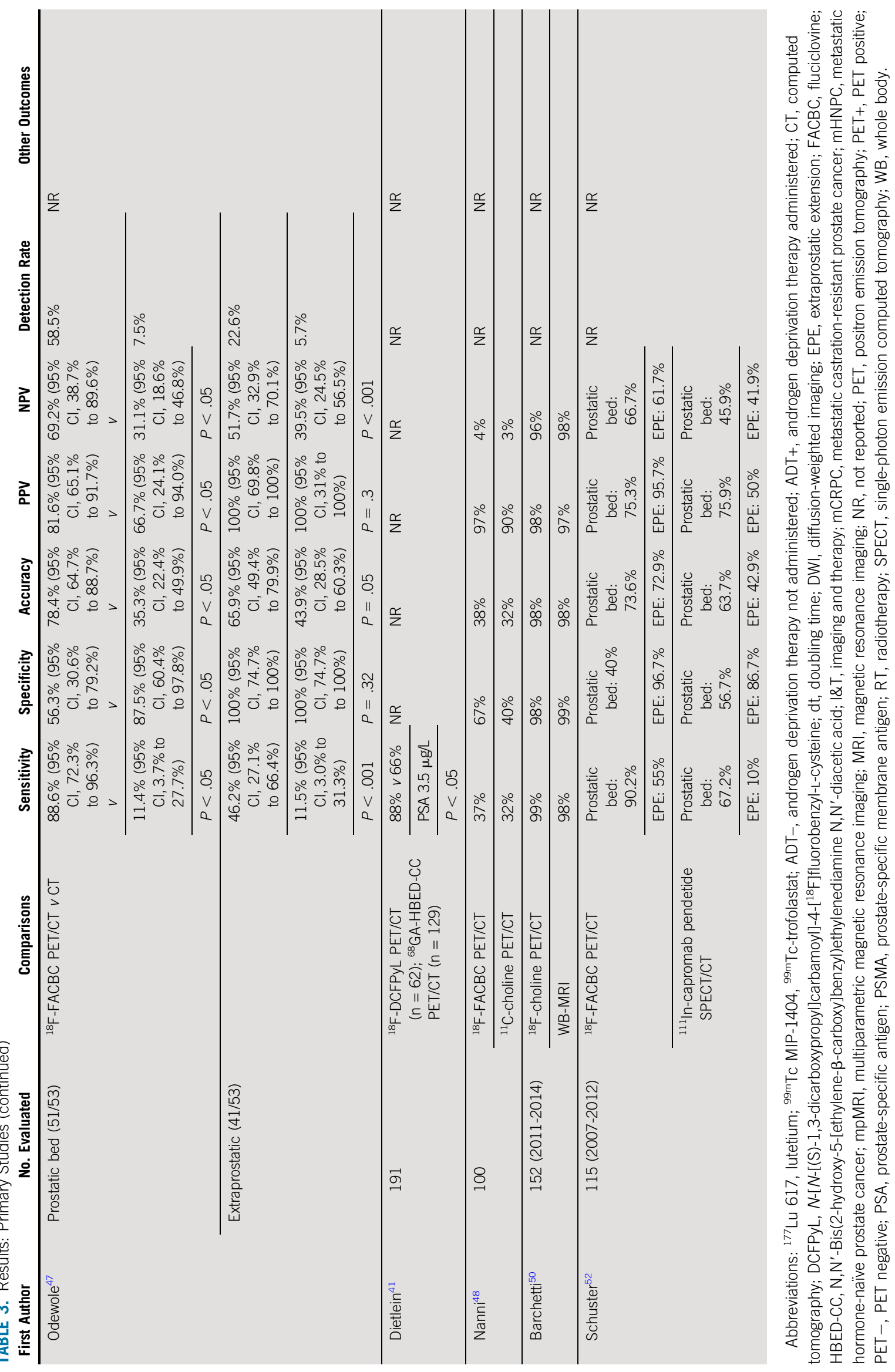


specificity of $93 \%(95 \% \mathrm{Cl}, 83 \%$ to $98 \%)$ for the detection of recurrent disease with ${ }^{11} \mathrm{C}$-acetate PET. It also found recurrence detection was higher for patients following surgery and/or radiotherapy and for patients with PSA at $>1 \mathrm{ng} / \mathrm{mL}$ at relapse.

- Umbehr et $\mathrm{al}^{29}$ in a systematic review of 44 studies including 2,293 patients in which 29 studies were pooled, found both ${ }^{11} \mathrm{C}$-choline PET and ${ }^{18} \mathrm{~F}$-choline $\mathrm{PET}$ to be useful in restaging patients with biochemical recurrence to determine the best treatment plan.

- In a systematic review of 15 studies involving 1,256 patients, von Eyben et al ${ }^{20}$ found ${ }^{68} \mathrm{Ga}-\mathrm{PSMA} \mathrm{PET} / \mathrm{CT}$ to have utility in the detection of disease recurrence for patients with rising PSA after radical prostatectomy with PSA levels $<1.0 \mathrm{ng} / \mathrm{mL}$.

- Fitzpatrick et al, ${ }^{18}$ in a systematic review of 24 studies involving 2,408 patients, found ${ }^{68} \mathrm{Ga}-\mathrm{PSMA} \mathrm{PET} / \mathrm{CT}$ associated with good sensitivity (33\% to 93\%) and high specificity (>99\%). The likelihood of detection was found to increase with rising PSA levels and at low PSA levels, was greater than that of current choline tracers. Early detection of recurrence does allow for changes to any follow-up treatment plan. The authors note that detection may be affected by tracer trapping, androgen deprivation therapy (ADT), and levels of PSMA expression.

- In a systematic review of 18 cohort studies involving 2,219 patients, von Eyben et al ${ }^{19}$ found no difference in detection rates between ${ }^{11} \mathrm{C}$-choline PET/CT and ${ }^{18} \mathrm{~F}-\mathrm{FCH} \mathrm{PET} / \mathrm{CT}$ in patients with suspected recurrence $(P=.26)$.

- Fanti et al, ${ }^{24}$ in a systematic review of 29 diagnostic studies involving 2,686 patients, found ${ }^{11} \mathrm{C}$-choline $\mathrm{PET} / \mathrm{CT}$ to have high accuracy and good sensitivity (89\%) and specificity (89\%) to detect local and/or distant recurrence in previously treated patients with prostate cancer.

- In a systematic of review of 19 studies involving 1,555 patients, Evangelista et a ${ }^{32}$ found both ${ }^{18} \mathrm{~F}$-choline PET or ${ }^{11} \mathrm{C}$-choline PET or PET/CT to have high pooled sensitivity $(81.8 \% ; 95 \% \mathrm{Cl}, 77.9 \%$ to $85.2 \%)$ and specificity $(91.4 \% ; 95 \% \mathrm{Cl}, 88.3 \%$ to $93.9 \%)$ to detect local and/or distant recurrence in previously treated patients with prostate cancer.

- In a systematic review of 16 studies involving 1,447 patients (including 23 controls), Alfarone et a ${ }^{34}$ found multiparametric MRI ( $m p M R I)$ superior to ${ }^{18} \mathrm{~F}$-choline or 11C-choline PET/CT in detecting local recurrence in patients with low PSA and with small-diameter lesions.

- Ren et al, ${ }^{21}$ in a systematic review of 6 studies involving 251 patients, found ${ }^{18} \mathrm{~F}$-fluciclovine PET/CT to have high sensitivity (88\%) and acceptable specificity (67\%) to detect prostate cancer recurrence.

- Shen et al, ${ }^{27}$ in a systematic review of 18 studies involving 1,102 patients, found MRI superior to ${ }^{11} \mathrm{C}$-choline PET/CT, SPECT, and bone scintigraphy in the detection of bone metastases on a per-patient basis but found PET/CT to be superior to bone SPECT and bone scintigraphy on a per-lesion basis, with a higher diagnostic odds ratio and maximum sensitivity and specificity scores $\left(Q^{*}\right)$.

- In a systematic review of 7 studies involving 603 patients, Silva et $a^{31}$ found 1.5-T MRI to have low sensitivity (49\%) and specificity (58\%) when used for the diagnosis and staging of prostate cancer but that specificity increased when used to determine seminal vesicle invasion (96\%) and extraprostatic extension (82\%), while sensitivity remained low for both $(45 \%$ and $49 \%$, respectively).

- In a systematic review of 18 studies (10 of which were pooled) involving 441 patients, Evangelista et al ${ }^{33}$ found ${ }^{11} \mathrm{C}$-choline PET/CT or ${ }^{18} \mathrm{~F}$-choline PET/CT to have low pooled sensitivity $(0.43 ; 95 \% \mathrm{Cl}, 0.33$ to $0.53)$ but high specificity $(0.95 ; 95 \% \mathrm{Cl}, 0.91$ to 0.97$)$ in the detection of lymph node metastases prior to surgery in patients with prostate cancer.

- In a systematic review of 75 studies involving 5,681 patients, de Rooij et al ${ }^{25}$ found MRI (diffusion-weighted [DW] imaging, dynamic contrast enhanced [DCE]) to have high specificity but low sensitivity for staging local prostate cancer. Use of an endorectal coil demonstrated no increase in detection rates for extracapsular extension but did demonstrate higher sensitivity for detecting seminal vesicle involvement.

- In a systematic review of 16 studies involving 1,309 patients, Perera et $\mathrm{al}^{22}$ found ${ }^{68} \mathrm{Ga}$-PSMA PET to have superior sensitivity and specificity compared with choline-based PET imaging options and that rising PSA values and positive ${ }^{68} \mathrm{Ga}-\mathrm{PSMA}$ PET results are correlated.

Primary research articles. Eighteen primary research articles ${ }^{35-52}$ were obtained. Full details are provided in Table 3:

- Three studies ${ }^{35,36,38}$ investigated the role of the PSMA binding ligand ${ }^{99 \mathrm{~m} T \mathrm{~T}-M I P-1404}$ imaged with wholebody planar and SPECT/CT compared with histology, and one of these ${ }^{38}$ compared the results of both against MRI findings. The detection rates with ${ }^{99 \mathrm{~m} T c-}$ MIP-1404 whole-body planar and SPECT/CT were found to vary with PSA level in one study, ${ }^{36}$ with higher PSA being associated with higher detection rates. In this same study, ADT was also associated with higher detection rates (ADT positive, 86\%; ADT negative, $71 \% ; P<.001$ ). The study that compared ${ }^{99 \mathrm{~m} T \mathrm{TC}-\mathrm{MIP}-}$ 1404 SPECT/CT with MRI found higher detection rates for SPECT/CT (SPECT/CT, 94\%; MRI, 86\%). ${ }^{38}$

- Seven $37,39,40,42,43,46,51$ studies reported on PSMA binding ligand ${ }^{68} \mathrm{Ga}$ PET/CT. Two ${ }^{40,43}$ reported on PSMA binding ligand ${ }^{68} \mathrm{Ga}$ imaged with $\mathrm{PET}$, and five $^{37,39,42,46,51}$ reported on PSMA binding ligand ${ }^{68} \mathrm{Ga}$ imaged with PET in comparison with PET/CT, 
PET/MRI, or mpMRI. Einspieler et al, ${ }^{40}$ in a study of the PSMA binding ligand ${ }^{68} \mathrm{Ga}$ imaged with PET/CT, found that the detection rate increased with both PSA and with ADT (PSA 2 to $<5 \mathrm{ng} / \mathrm{mL}, 81.8 \% \mathrm{v}$ PSA 5 to $<10 \mathrm{ng} / \mathrm{mL}, 95.3 \% \vee$ PSA $\geq 10 \mathrm{ng} / \mathrm{mL}$, 96.8\%; $P=.038$ ) and ADT (ADT positive, $97.7 \% \mathrm{v}$; ADT negative, $86.3 \% ; P=.038$ ). Two studies 37,43 reported that changes to the treatment plan were made based on the results, with the study by Albisinni et al ${ }^{43}$ reporting changing $76 \%$ (99 of 131) of the planned treatments and the study by $\mathrm{Habl}$ et $\mathrm{a}^{37}$ reporting changing the staging of $43 \%$ of patients and $59 \%$ of patients having a change made to the radiation treatment plan due to ${ }^{68} \mathrm{Ga}$-PSMA PET/CT outcomes. For detection of prostate cancer, the study reported by Freitag et $\mathrm{al}^{39}$ found equivalent rates between ${ }^{68} \mathrm{Ga}-$ PSMA-11 PET/CT and ${ }^{68}$ Ga-PSMA-11 PET/MRI (PET/ CT, 7.6\%; PET/MRI, 7.6\%) but found both to be inferior to $\mathrm{mpMRI}(15.1 \% ; P=.004)$. The two ${ }^{68} \mathrm{Ga}-\mathrm{HBED}-\mathrm{CC}$ studies reported by Berliner et $\mathrm{al}^{42}$ and Ceci et $\mathrm{al}^{51}$ both found that detection rates increased with PSA. For Berliner et al, this difference was significant at all PSA levels $(P<.05)$, and for Ceci et al, while reporting an overall detection rate of $74.2 \%$, having a positive PET scan was associated with higher PSA rates and faster PSA doubling times, and negative PET scans were associated with the reverse. Of these 7 studies, only Pfister et a ${ }^{46}$ reported on measures of diagnostic utility with ${ }^{68} \mathrm{Ga}-\mathrm{HBED}-\mathrm{CC}$ being associated with higher sensitivity (86.9\% $\vee 71.2 \%)$, specificity $(93.1 \% \vee$ $86.9 \%)$, accuracy $(91.9 \% \vee 82.5 \%)$, positive predictive value (PPV; 75.7\% $\vee 67.3 \%$ ), and negative predictive value (NPV; $96.6 \%$ v 88.8\%) compared with ${ }^{18} \mathrm{~F}$-fluoroethylcholine PET/CT, but only accuracy and NPV were statistically different at $P<.05$.

- One study, reported by Rahbar et $a l,{ }^{45}$ examined radionuclide therapy with the PSMA binding ligand ${ }^{177} \mathrm{Lu}$ 617 followed by ${ }^{68} \mathrm{Ga}$-PSMA PET to confirm PSMA expression and found that $64 \%$ of patients (53 of 82) experienced a PSA decline (31\% experienced a $>50 \%$ decline in PSA).

- One study, reported by Larbi et al, ${ }^{49}$ examined wholebody MRI/DW imaging in the detection of oligometastatic disease (defined in that study as $\leq 3$ synchronous lesions) for treatment planning and found $28 \%$ of all patients with metastatic and hormone-naive prostate cancer were oligometastatic and that $50 \%$ of patients with metastatic CRPC were oligometastatic.

- $\mathrm{Six}^{41,44,47,48,50,52}$ reported on ${ }^{18} \mathrm{~F}$ with various radiotracers against other modalities. The study by AkinAkintayo et $\mathrm{al}^{44}$ in a comparison of ${ }^{18} \mathrm{~F}$-fluciclovine $\mathrm{PET} / \mathrm{CT}$ pre- and post-radiation treatment decision, reported a detection rate of $81 \%$, while $40.5 \%$ of patients experienced a change in their treatment plan after PET/CT $(P<.001)$. The study by Odewole et al, ${ }^{47}$ in a comparison between ${ }^{18} \mathrm{~F}$-fluciclovine PET/CT and
CT alone, reported a detection rate of $77.4 \%$ with PET/ CT versus $18.9 \%$ with CT alone $(P<.05)$. Five studies reported on measures of diagnostic utility. The study by Odewole et $\mathrm{al}^{47}$ in a comparison between ${ }^{18} \mathrm{~F}$-fluciclovine PET/CT and CT alone, found significantly higher sensitivity, accuracy, PPV, and NPV (all $P<.05$ ) associated with ${ }^{18} \mathrm{~F}$-fluciclovine PET/CT in the prostate/bed, while CT alone reported significantly higher specificity in the prostate/bed. For extraprostatic disease, ${ }^{18} \mathrm{~F}$-fluciclovine PET/CT in comparison with $\mathrm{CT}$ alone had significantly higher sensitivity and NPV $(P<.05)$, borderline higher accuracy $(P=.05)$, and similar high specificity and PPV of $100 \%$, respectively. The study by Dietlein et $\mathrm{al}^{4}{ }^{41}$ in a comparison between 2 PSMA binding ligands ( ${ }^{18} \mathrm{~F}$-DCFPyL PET/CT and ${ }^{68}$ GA-HBED-CC PET/CT), reported sensitivity of $88 \%$ with ${ }^{18} \mathrm{~F}$-DCFPyL PET/CT $v 66 \%$ with ${ }^{68} \mathrm{Ga}-H B E D-C C$ PET/CT for PSA values $<3.5 \mu \mathrm{g} / \mathrm{L}$ $(P<.05)$. The study by Nanni et al, ${ }^{48}$ in a comparison between ${ }^{18} \mathrm{~F}$-fluciclovine PET/CT and ${ }^{11} \mathrm{C}$-choline PET/ $C T$, reported superior sensitivity, specificity, accuracy, PPV, and NPV associated with fluciclovine PET/CT over choline PET/CT ( $P$ values not reported). In a comparison between ${ }^{18} \mathrm{~F}$-choline PET/CT and wholebody MRI, Barchetti et al ${ }^{50}$ found equivalent sensitivity, specificity, accuracy, PPV, and NPV. Schuster et al, ${ }^{52}$ in a comparison between ${ }^{18} \mathrm{~F}$-fluciclovine PET/CT and ${ }^{111}$ In-capromab pendetide SPECT/CT for measures of diagnostic utility in scans of the prostatic bed and extraprostatic extension, found ${ }^{18} \mathrm{~F}$-fluciclovine PET/ CT superior to ${ }^{111}$ In-capromab pendetide SPECT/CT for sensitivity and accuracy, and without significant differences for specificity, PPV, and NPV for scans of the prostatic bed; and found ${ }^{18} \mathrm{~F}$-fluciclovine PET/CT superior to ${ }^{111}$ In-capromab pendetide SPECT/CT for sensitvity, accuracy, PPV, and NPV, and equivalent high specificity for scans of extraprostatic extension.

\section{RECOMMENDATIONS}

\section{CLINICAL QUESTION 1}

What is the goal of imaging in advanced prostate cancer?

\section{Recommendation 1}

Imaging is recommended for all patients with advanced prostate cancer. See the recommendation under clinical question 4 for specific details according to clinical scenario (Type: evidence based, benefits outweigh harms; Evidence quality: moderate; Strength of recommendation: strong).

Literature review, analysis, and clinical interpretation. All the evidence obtained in this clinical practice guideline (17 systematic reviews with or without meta-analysis and 18 primary articles) supported the use of imaging in advanced prostate cancer. The goal of imaging in advanced prostate cancer is to facilitate the accurate and timely detection and localization of sites of prostate cancer spread and extent, 
thus contributing to the decision-making process for treatment planning, follow-up, and response assessment.

\section{CLINICAL QUESTION 2}

What imaging techniques are available for imaging advanced prostate cancer?

\section{Recommendation 2}

One or more of the following imaging modalities should be used for patients with advanced prostate cancer: conventional imaging (defined as CT, bone scan, and/or prostate MRI) and/or NGI (PET, PET/CT, PET/MRI, wholebody MRI), according to clinical scenario (Type: evidence based, benefits outweigh harms; Evidence quality: moderate; Strength of recommendation: strong).

Literature review, analysis, and clinical interpretation. Plain film/CT. Plain film radiography relies on the differential penetration of ionizing radiation ( $\mathrm{x}$-rays) through body tissues of variable density to produce a 2-dimensional representation of part of the anatomy. Its use in prostate cancer is limited, including assessment of questionable bone scan findings as a crude measure of bony metastasis, as well as for rapid assessment of complications (eg, bone fractures, pneumonia). Although large bone metastases can be visible on plain radiography, the modality plays a limited role in achieving the presented goals of imaging in advanced prostate cancer.

CT also relies on $\mathrm{x}$-rays but takes advantage of computational postprocessing tools to generate high-resolution 3-dimensional images with exquisite anatomic detail. CT is extensively used in staging and response assessments of many cancers. Its main advantages are wide availability and rapid imaging acquisition (a whole-body scan can be performed in only a few seconds). The main limitations in advanced prostate cancer are (1) relatively poor performance for detection of bone metastases, as a substantial amount of cortical destruction is necessary before bone lesions are visible on $\mathrm{CT}$, and thus early metastases are not detected, and (2) limited assessment of lymph node metastases primarily due to heavy reliance on morphologic features (eg, size, shape, borders). CT is unable to identify micrometastases in normal-sized nodes and unable to accurately distinguish enlarged hyperplastic (benign) from malignant nodes. CT is useful for the assessment of questionable bone scan findings by demonstrating benign conditions (trauma, degenerative changes) that result in false-positive appearances on bone scan.

MRI. MRI produces multiplanar images without the need for potentially harmful ionizing radiation. The strong magnetic field in the bore of an MRI scanner causes protons (which are naturally abundant in the human body) to align in its direction. A radiofrequency (RF) pulse is then applied to "spin" the body's protons out of equilibrium. Advanced computational tools are then used to generate images based on the data collected, including the amount of energy released and the time it takes protons to "realign" with the magnetic field once the RF pulse is turned off. Multiple imaging acquisition parameters can be modified to provide different "weighting" of the images (eg, T1, T2 weighting), each providing different degrees of visualization representing different physical and biologic properties of tissues. Compared with CT, MRI is particularly useful in the assessment of the bone marrow, as bone marrow edema resulting from early seeding of metastatic cancer foci, not visible on $\mathrm{CT}$, can be depicted on MRI. MR also superbly delineates the prostatic zonal anatomy not clearly visualized on other modalities and has thus firmly established itself as the imaging tool of choice for the assessment of primary prostatic tumors, using a multiparametric acquisition approach combining "anatomic" (T1- and T2-weighted) and "functional" (DW and DCE) sequences. Limitations of MRI include longer examination times compared with CT (pelvic MRI typically 20-30 minutes; whole-body MRI approximately twice as long); interpretation expertise not as widely available; and shortcomings with regard to lymph node metastases, which also rely heavily on the same morphological features assessed with CT. Due to increasing evidence of gadolinium deposition in normal tissues (eg, brain) and lack of knowledge of the long-term health implications of this finding, it is widely advocated that intravenous gadolinium for MRI is only used when potential benefits outweigh risks.

Improvements in diagnostic performance for detection of lymph node metastasis can be obtained by using nomograms that consider the pretest probability based on the risk status of the primary tumor and local staging information. ${ }^{51}$ Taken together, the general test performance of morphologic imaging remains limited when histologic correlations using template lymphadenectomy is used as the standard of reference; a recent meta-analysis showed a CT scan sensitivity of $42 \%(95 \% \mathrm{Cl}, 20 \%$ to $56 \%$ ) and specificity of $82 \%(95 \% \mathrm{Cl}, 80 \%$ to $83 \%)$, while MRI had a sensitivity of $39 \%(95 \% \mathrm{Cl}, 19 \%$ to $56 \%)$ and specificity of $82 \%(95 \%$ $\mathrm{Cl}, 79 \%$ to $83 \%){ }^{52}$ Thus, morphologic CT and MRI misrepresent nodal status and can misdirect the therapeutic approach. There have been concerted efforts to improve metastatic nodal detection test performance, and one promising technique is ferumoxtran-10-enhanced MRI, which has been shown to be able to detect microscopic nodal disease. ${ }^{53}$ Unfortunately, lack of general commercial availability and regulatory approval makes this method of assessment unobtainable for most.

Bone scintigraphy. Bone scans date back to the 1960s. Over the course of time, the technique found favor for being relatively inexpensive and capable of whole-body assessment. It involves administration of a diphosphonate, which mimics phosphate in bone mineral and adsorbs to areas of active bone formation, particularly around metastases where osteoblastic activity is prominent. In some cases, it can detect lesions not seen by CT. ${ }^{54-56}$ However, there are 
numerous disadvantages. As the bone scan images not the tumor itself but the body's response to it, new bone formation in response to tumor responding to therapy can appear as a new lesion (flare response). ${ }^{57,58}$ As a result, Prostate Cancer Working Group 3 guidelines require new bone lesions to be seen on two consecutive scans, and new lesions to be seen on the second scan, before progression can be called. ${ }^{4}$ Although this approach leads to a reliable diagnosis of bone metastases, there is an inherent delay in establishing this due to the need for confirmatory scans. It can also be difficult to quantify disease and therefore track progression. A quantitative measure of involved bone, the BSI, has been developed, and correlates with overall survival; however, its validity as a biomarker is still under study. ${ }^{59}$ A normal bone scan produces two-dimensional images; the three-dimensional imaging option, SPECT, is very slow, only allowing imaging of perhaps one or two anatomic segments of the body, such as the chest and abdomen or abdomen and pelvis, over the course of 30 minutes (compare this with a PET scanner, which can scan the whole body in about 15 to 20 minutes or less). Finally, of course, a bone scan only examines the bones and will ignore lymphatic or visceral metastases detected by $\mathrm{CT}$, MRI, or PET.

There is a large generic body of evidence for the use of bone scan in prostate cancer. A recent meta-analysis looked specifically at the yield of bone scintigraphy in the initial staging of treatment-naive prostate cancer, and found a (relatively low) yield of $3.5 \%$ with PSA $\leq 10,6.9 \%$ with $10<$ PSA $\leq 20$, and $41.8 \%$ with PSA $>20$ over 54 studies. ${ }^{60}$ Detection rate is similarly $4.1 \%$ with Gleason score $\leq 6,10 \%$ with Gleason score 7, and $28.7 \%$ with Gleason score $\geq 8$. This suggests that the existing practice of using bone scan specifically for initial staging of higher-risk patients (and not using it for lower-risk patients) is sound.

PET and PET/CT. PET/CT with a variety of radiopharmaceuticals has received much attention in oncology over the past several years in alleviating many of the limitations of standard imaging methods. The radiopharmaceutical used in PET consists of a pharmaceutical agent or "tracer" (with biologic properties [eg, receptor binding] determining its site of accumulation in the body) labeled with a positron emitting radioisotope (eg, ${ }^{18} \mathrm{~F}$ [absorption half-life $\left[\mathrm{t}_{1 / 2}\right]$, 110 minutes], ${ }^{11} \mathrm{C}\left[\mathrm{t}_{1 / 2}, 20\right.$ minutes $],{ }^{68} \mathrm{Ga}\left[\mathrm{t}_{1 / 2}, 68 \mathrm{~min}\right.$ utes]), which will allow its detection with a PET camera. FDG labeled with the isotope ${ }^{18} \mathrm{~F}$ is the most common PET radiopharmaceutical used in oncology. FDG accumulation in tumors is related to elevated glucose metabolism in malignant tissue. In prostate cancer, the diagnostic performance of FDG PET/CT is highly dependent on the phase of the disease. Cumulative current evidence suggests that FDG PET may be useful in the imaging evaluation of extent and treatment response in metastatic castrationresistant disease ${ }^{61}$ but not in localized prostate cancer or in early noncastrate metastatic states. Lipogenesis radiopharmaceuticals, including ${ }^{11} \mathrm{C}$-acetate and ${ }^{18} \mathrm{~F}$ - or ${ }^{11} \mathrm{C}$-labeled choline, have also been investigated relatively extensively. Most studies, primarily from Europe and Japan, with choline-based radiotracers have focused on the biochemical recurrence of disease. ${ }^{11} \mathrm{C}$-choline was approved in the United States in 2012 for imaging evaluation of men with biochemical recurrence of prostate cancer after definitive primary therapy. More recently in 2016, the amino acid analog PET radiotracer ${ }^{18} \mathrm{~F}$-fluciclovine was also approved in the United States for imaging evaluation of men with biochemical recurrence of prostate cancer. There are several other unapproved PET radiotracers that are actively being investigated in the imaging evaluation of prostate cancer. Of these radiotracers, those targeting the PSMA receptor have received much attention with exciting results. PSMA is a transmembrane protein expressed in the secretory cells of the prostate epithelium as well as nonprostate normal and malignant tissues. In prostate cancer, the PSMA cleavage of vitamin B9 (folic acid) stimulates oncogenic signaling through glutamate receptors with downstream activation of the PI3K-Akt-mTOR signaling pathway. ${ }^{62}$ Recent strides in synthesis of small-molecule inhibitors of PSMA targeting the extracellular epitope of PSMA have demonstrated major potential utility in targeted radionuclide imaging and treatment (theranostics) of metastatic prostate cancer. Most studies have reported on ${ }^{68}$ Ga-PSMA-11 (also known as HBED-CC). Other PSMA binding ligands include ${ }^{68} \mathrm{Ga}-\mathrm{PSMA}$ imaging and therapy; ${ }^{68} \mathrm{Ga}-\mathrm{PSMA}-617$; and more recently, ${ }^{18} \mathrm{~F}$-DCFPyL and ${ }^{18}$ F-PSMA-1007. ${ }^{63,64}$ Studies have generally shown superior diagnostic performance of these radiotracers over other relevant radiotracers in the clinical settings of intermediateto high-risk primary cancer, biochemical recurrence after definitive therapy, and delineation of extent of metastatic disease and patient eligibility for PSMA-targeted radioligand therapy. ${ }^{22}$ 89-Zr-labeled PSMA-targeting antibodies and minibodies have also been reported, but practicalities surrounding their use (eg, serial days uptake required between administration and imaging) have resulted in their development being largely restricted to tertiary academic centers. It must be noted that false-negatives and falsepositives can occur with PSMA PET imaging. ${ }^{65}$ There is also literature on proposed procedure guidelines and interpretation and reporting standards. ${ }^{66-70}$ A number of studies have reported on the major impact of PSMA PET imaging on management of patients with prostate cancer, although the potential influence on outcome will need additional investigations. ${ }^{71-73}$ It is hoped that of the several PSMA-based imaging agents that have been evaluated, the most optimal agent will emerge that will become approved, available, and accessible.

In general, radiocholine may be useful in this clinical setting when PSA $>2 \mathrm{ng} / \mathrm{mL}$ (or PSA $<1 \mathrm{ng} / \mathrm{mL}$ if primary Gleason score was 7or more), PSA doubling time is less than 6 months, or PSA velocity is greater than $2 \mathrm{ng} / \mathrm{mL} /$ year. 
Higher serum PSA level during biochemical recurrence is generally associated with higher detectability on choline PET, with a pooled sensitivity and specificity of $80.9 \%$ and $84.1 \%$, respectively. ${ }^{74}$ The diagnostic performance of ${ }^{18} \mathrm{~F}$-fluciclovine in the detection of potential lesions is also positively associated with PSA level. In the clinically relevant low PSA range of $<1 \mathrm{ng} / \mathrm{mL}$ in men with biochemical recurrence of prostate cancer after radical prostatectomy and off hormonal therapy, the detectability of ${ }^{18} \mathrm{~F}$-fluciclovine PET has been reported to be $21 \%$ in comparison with $14 \%$ with ${ }^{11} \mathrm{C}$-choline. ${ }^{48}$ However, in general, these two approved radiotracers appear to be similar in terms of lesion detectability and positive correlation with serum PSA level in the clinical setting of biochemically recurrent prostate cancer. ${ }^{75}$

PET/MRI. PET/CT has dominated the molecular imaging landscape for almost two decades as it combines the sensitivity and specificity of PET and the anatomic depiction of CT. PET/MRI devices have been available since 2010 and have been tried in a broad range of clinical settings. However, in general, they have not provided a distinct advantage over PET/CT beyond the simultaneous acquisition of diagnostic PET and MRI when both tests are clinically indicated. PET/MRI can be helpful in specific cases. In theory, PET/MRI devices have several advantages over PET/CT, including that MRI provides not only anatomy but also functional imaging information that could increase the specificity of PET findings, and in several regions, such as the pelvis and bones, MRI provides additional information that could help to characterize a lesion with PET uptake.

In the initial diagnosis and biopsy of primary prostate cancer, numerous studies have documented an advantage to tumor localization using a combination of PET and MRI either at separate settings or obtained simultaneously in PET/MRI scanners. ${ }^{76}$ The most documented advantage is found for PSMA PET imaging in which numerous studies document improvement in detection when combining PET and $\mathrm{MRI},{ }^{76-81}$ although similar findings have been noted with fluciclovine. ${ }^{82-84}$ Not all studies are positive, and PET/MRI with ${ }^{11} \mathrm{C}$-choline ${ }^{76,85}$ and one study of PSMA ${ }^{86}$ showed no advantage to PET/MRI. Occasionally, MRI is negative, but PET, particularly with PSMA-targeted agents, is positive, ${ }^{78,81}$ aiding in diagnosis. On the other hand, PSMA-negative primary tumor occurs in up to $5 \%-10 \% .{ }^{87} \mathrm{MRI}$ also provides better evaluation of local staging in high-risk patients, such as extraprostatic extension or seminal vesicle invasion, than can PET/CT due to superior spatial and contrast resolution.

In biochemical recurrence, the ability to document sites of localized recurrence ( $v$ nodal or bony disease) is perceived as a potential advantage for PET/MRI across many agents. ${ }^{63,76,77,80}$ Particular benefits of MRI versus CT include absence of clip artifacts from prior node dissections and absence of streaking artifacts from dense contrast media within the bladder that can interfere with $\mathrm{CT}$ scans.
In metastatic disease, there is a perceived advantage of whole-body MRI in detecting bone marrow changes ${ }^{76,81}$ based on the sensitivity of DW-MRI for subtle bone disease and the high contrast of T1-weighted sequences for bone marrow replacement. However, few studies of prostate cancer with PET/MRI have been performed, and so there is an absence of strong data in this setting. For metastatic disease, whole-body imaging sequences can add considerable time to the scan. Thus, such whole-body imaging is limited to one sequence (eg, DW or T2-weighted turbo spin echo) and/or postcontrast-enhanced MRI, which are the most time efficient methods of evaluating the bones in conjunction with PET. However, this causes a significant increase in scanning time. Thus, while the increased sensitivity of MRI for bone lesions could be helpful in patients with suspected metastatic disease, especially with a negative $\mathrm{CT}$, clinical trial evidence is not yet available to support this.

The advantages of PET/MRI must be weighed against multiple disadvantages. The cost of such hybrid devices is approximately 2-3 times that of a conventional PET/CT. Cost recovery is more difficult as the scans take longer to obtain, reducing throughput compared with PET/CT. Additional unresolved issues include the accuracy of standardized uptake value measurements on PET/MRI compared with PET/ CT. Moreover, it is unclear whether it is vital that MRI and PET be obtained simultaneously in a PET/MRI hybrid scanner or can be obtained separately as a PET/CT and a dedicated MRI. It is possible that a PET/CT and a stand-alone MRI could be performed and achieve the same advantages without the cost of an expensive hybrid scanner. ${ }^{85,88}$

Capromab pendetide. Capromab pendetide is an ${ }^{111} \mathrm{In}$ labeled antibody directed against the intracellular epitope of PSMA. It is imaged using SPECT/gamma cameras and is currently approved for initial staging of high-risk patients and in localization of recurrence after biochemical failure. Due to the difficulties of antibody imaging (imaging is slow, occurring 3-5 days after injection, and burdened by physiologic and generic antibody uptake in the bone marrow, liver, and spleen) and the poor sensitivity due to the intracellular localization of the epitope (making it unable to visualize living cancer cells, particularly in bone), the study is rarely performed at present. Among the few studies comparing capromab pendetide directly to other imaging modalities (no meta-analyses exist), one of 93 patients found it inferior to the new agent fluciclovine at $10 \%$ sensitivity and $87 \%$ specificity for extraprostatic disease versus $55 \%$ sensitivity and $97 \%$ specificity ${ }^{48}$ for recurrence. A similar study with 50 patients showed $10 \%$ sensitivity and $100 \%$ specificity versus fluciclovine with $100 \%$ sensitivity and specificity for extraprostatic disease. ${ }^{89}$ An old study comparing capromab pendetide to FDG PET and CT showed lower detection rates, although sample size was very small $\left(n=21\right.$ patients). ${ }^{90}$ 


\section{CLINICAL QUESTION 3}

What are the unmet needs and potential impact of imaging according to different prostate cancer disease states?

\section{Recommendation 3}

It is recommended when choosing an imaging modality that disease states and clinical scenarios as outlined are taken into consideration, as the imaging modality may guide treatment or change clinical treatment decisions (Type: evidence based, benefits outweigh harms; Evidence quality: moderate; Strength of recommendation: strong).

Literature review, analysis, and clinical interpretation. Prostate cancer frequently recurs despite negative conventional imaging, presumably partly due to unrecognized residual disease after treatment or distant metastatic disease. For this reason, there is an unmet need for accurate diagnosis of metastatic disease for accurate staging, appropriate counseling, and adequate treatment planning for all stages of the disease. Improved and more accurate diagnosis of disease outside of the prostate or subclinical metastatic disease offers the potential to modulate or change treatment. More accurate staging at initial diagnosis could influence the local therapy offered (surgery, radiotherapy, ablation, surveillance, and so on). For initial surgery, imaging can impact planning of nerve-sparing procedures and help to determine the extent of lymphadenectomy. For radiotherapy, the duration of concurrent and adjuvant ADT could be impacted as well as the extent of radiation field (whole pelvic to include nodal drainage or just prostate). At the time of biochemical recurrence after local therapy, the aggressiveness and targeting of salvage local therapy could also be impacted. For patients with advanced disease, the timing of changes in systemic therapies and the accurate ability to monitor response to therapy could be impacted by more accurate imaging.

\section{CLINICAL QUESTION 4}

When and what type of imaging is appropriate in each scenario?

\section{Newly Diagnosed Clinically High-Risk/Very High-Risk Localized Prostate Cancer}

Recommendation 4.1. Conventional imaging negative When conventional imaging (defined as CT, bone scan, and/or prostate MRI) is negative in patients with a high risk of metastatic disease, NGI (defined as PET, PET/CT, PET/ MRI, whole-body MRI) may add clinical benefit, although prospective data are limited (Type: informal consensus, benefits/harm ratio uncertain; Evidence quality: weak; Strength of recommendation: moderate).

\section{Recommendation 4.2. Conventional imaging suspicious/ equivocal}

When conventional imaging is suspicious or equivocal, NGI may be offered to patients for clarification of equivocal findings or detection of additional sites of disease, which could potentially alter management, although prospective data are limited (Type: informal consensus, benefits/harm ratio uncertain; Evidence quality: weak; Strength of recommendation: moderate).

Literature review, analysis, and clinical interpretation. Patients presenting with high-risk, including locally advanced, prostate cancer have a high probability of harboring metastatic disease. The prevalence of metastases is reported to be between $30 \%$ and $50 \%$ of patients, depending on the sensitivity of the method used for disease detection. ${ }^{91}$ Metastases are most commonly located within regional pelvic lymph nodes and in bone, with metastases located at other distant sites being rare at this stage of the disease.

There is wide geographic variation in the availability of NGI technologies (whole-body MRI and PET/CT), but when available, they should be considered in the context of a clinical trial so that generalizable data can be recorded. For the detection of metastatic bone disease, the combination of bone scans and CT perform suboptimally compared with whole-body MRI and various PET/CT radiopharmaceuticals. ${ }^{92}$ Systematic analyses, prospective clinical studies, and meta-analyses have shown comparative test performance of whole-body MRI to NaF and choline PET/CT for the skeletal assessments in advanced prostate cancer. ${ }^{27,84}$ Shen et al ${ }^{27}$ conducted a meta-analysis of 27 studies in advanced prostate cancer showing MRI was superior to choline PET/CT and bone scan for metastasis detection on a per-patient basis. On a per-patient basis, the pooled sensitivities for bone disease by using choline PET/CT, whole-body MRI, and bone scan were $91 \%(95 \% \mathrm{Cl}, 83 \%$ to $96 \%), 97 \%(95 \% \mathrm{Cl}$, $91 \%$ to $99 \%$ ), and $79 \%$ (95\% $\mathrm{Cl}, 73 \%$ to $83 \%$ ), respectively. The pooled specificities for bone metastases detection using choline PET/CT, whole-body MRI, and bone scan were $99 \%$ (95\% Cl, $93 \%$ to $100 \%$ ), $95 \%$ (95\% Cl, $90 \%$ to $97 \%$ ), and $82 \%(95 \% \mathrm{Cl}, 78 \%$ to $85 \%$ ), respectively. On a per-lesion analysis, choline PET/CT had a higher diagnostic odds ratio that exceeded both bone scan and bone SPECT for detecting bone metastases. A recent meta-analysis also underscored the usefulness of DW-MRI in detecting bone metastases. Liu et $a^{93}$ evaluated 32 studies with 1,507 patients and showed a pooled sensitivity, specificity, and area under the curve for DW-MRI of $95 \%$ ( $95 \% \mathrm{Cl}, 90 \%$ to $97 \%$ ), $92 \%$ (95\% Cl, 88\% to $95 \%$ ), and 0.98 , respectively, on a per-patient basis, and $91 \%$ (95\% Cl, $87 \%$ to $94 \%$ ), $94 \%$ (95\% Cl, $90 \%$ to $96 \%$ ), and 0.97 , respectively, on a per-lesion basis. This was recently confirmed by a prospective clinical trial where wholebody MRI was compared with $\mathrm{NaF}$ PET/CT. ${ }^{84}$

Suspicious findings on NGl would influence treatment decisions in patients with advanced prostate cancer and negative conventional imaging, opening the scope for multimodality treatment of primary and oligometastatic disease or systemic therapy for more extensive metastatic states. When evaluating the results of the meta-analyses, and indeed in all studies reporting test performance of imaging studies with any modality, it should be noted that there are intrinsic verification biases that are particularly 
prevalent at lesion-level analyses because it is simply not possible to obtain histopathology for every bone lesion detected for ethical/practical clinical reasons. As a result, most studies use combinations of imaging methods and/or follow-up as the standards of reference..$^{84,94}$ Furthermore, as with all external imaging methods, microscopic metastasis are unlikely to be detected so that true-negative rates are difficult to ascertain.

When conventional imaging is suspicious or equivocal for nodal or visceral lesions in castration-sensitive patients at the highest risk of metastatic disease, there is no clear consensus or level 1 evidence to support ${ }^{18} \mathrm{~F}$-FDG PET/CT and should not be routinely offered. Whole-body MRI and $\mathrm{NaF}$ PET/CT may offer clinical benefit in this scenario by redefining the true extent of disease and shifting treatment decisions accordingly, although prospective data are limited. ${ }^{95}$ There is limited experience with other PET and radionuclide agents, such as ${ }^{18} \mathrm{~F}$-fluciclovine and ${ }^{11} \mathrm{C}$ - and ${ }^{18} \mathrm{~F}$-choline, and they are not FDA approved in this setting. There is enthusiasm for the potential added value of PSMA PET/CT and PET/MRI for the assessment of the local and metastatic extent of prostate cancer in this context, ${ }^{80,96,97}$ although PSMA imaging is currently not FDA approved and should thus be only performed as part of clinical trials or other controlled research settings.

\section{Rising PSA After Prostatectomy and Negative Conventional Imaging (either initial PSA undetectable} with subsequent rise or PSA never nadirs to undetectable)

\section{Recommendation $\mathbf{4 . 3}$}

Both disease states are indicative of potentially undetected residual local, locoregional, or micrometastatic disease, and imaging options are not distinct or different between these scenarios. The goal of therapy and the potential use of salvage local therapies in these scenarios should guide the choice of imaging. For men who are not candidates or are unwilling to receive salvage local or regional therapy, additional NGI should not be offered (Type: informal consensus, benefits/harms ratio uncertain; Evidence quality: low; Strength of recommendation: moderate).

\section{Recommendation 4.4}

For men for whom salvage radiotherapy is contemplated, $\mathrm{NGI}$ should be offered (PSMA imaging [where available]; ${ }^{11} \mathrm{C}$-choline or ${ }^{18} \mathrm{~F}$-fluciclovine PET/CT; or PET/MRI, wholebody MRI, and/or ${ }^{18} \mathrm{~F}-\mathrm{NaF}$ PET/CT) as they have superior disease detection performance characteristics and may alter patient management (Type: evidence based, benefits outweigh harms; Evidence quality: high; Strength of recommendation: strong).

\section{Rising PSA After Radiotherapy and Negative Conventional Imaging \\ Recommendation 4.5}

For men in whom salvage local or regional therapy is not planned or is inappropriate, there is little evidence that NGI will alter treatment or prognosis. The role of $\mathrm{NGI}$ in this scenario is unclear and should not be offered, except in the context of an institutional review board-approved clinical trial (Type: informal consensus, benefits/harms ratio uncertain; Evidence quality: intermediate; Strength of recommendation: moderate).

\section{Recommendation 4.6}

For men for whom salvage local or regional therapy (eg, salvage prostatectomy, salvage ablative therapy, or salvage lymphadenectomy) is contemplated, there is evidence supporting NGI for detection of local and/or distant sites of disease. Findings on $\mathrm{NGI}$ could guide management in this setting (eg, salvage local, systemic or targeted treatment of metastatic disease, combined local and metastatic therapy). PSMA imaging (where available), ${ }^{11} \mathrm{C}$-choline or ${ }^{18} \mathrm{~F}$-fluciclovine PET/ CT or PET/MRI, whole-body MRI, and/or ${ }^{18} \mathrm{~F}-\mathrm{NaF}$ PET/CT have superior disease detection performance characteristics compared with conventional imaging and alter patient management, although data are limited (Type: evidence based, benefits outweigh harms; Evidence quality: intermediate; Strength of recommendation: moderate).

\section{Metastatic Prostate Cancer at Initial Diagnosis or After Initial Treatment, Hormone Sensitive}

\section{Recommendation 4.7}

In the initial evaluation of men presenting with hormonesensitive disease with demonstrable metastatic disease on conventional imaging, there is a potential role for NGI to clarify the burden of disease and potentially shift the treatment intent from multimodality management of oligometastatic disease to systemic anticancer therapy alone or in combination with targeted therapy for palliative purposes, but prospective data are limited (Type: informal consensus, benefits/harms ratio uncertain; Evidence quality: intermediate; Strength of recommendation: moderate).

\section{Recommendation 4.8. Nonmetastatic CRPC}

For men with nonmetastatic CRPC, NGI can be offered only if a change in the clinical care is contemplated. Assuming patients have received or are ineligible for local salvage treatment options, NGI may clarify the presence or absence of metastatic disease, but the data on detection capabilities of NGl in this setting and impact on management are limited (Type: consensus, benefits/harms ratio uncertain; Evidence quality: weak; Strength of recommendation: moderate).

\section{Recommendation 4.9. Metastatic CRPC (PSA progression)}

As recommended by the Prostate Cancer Working Group 3 consensus statements, ${ }^{98}$ PSA progression alone for men on treatment of metastatic CRPC should not be the sole reason to change therapy. Conventional imaging can be used for initial evaluation of PSA progression and should be continued to facilitate changes/comparisons and serially to assess for development of radiographic progression. (Type: informal consensus, benefits/harms ratio uncertain; Evidence quality: intermediate; Strength of recommendation: strong). 


\section{Recommendation 4.10}

The use of NGI in this cohort is unclear, with a paucity of prospective data. When a change in clinical care is contemplated, in an individualized manner, and there is a high clinical suspicion of subclinical metastasis despite negative conventional imaging, the use of NGl could be contemplated, especially in the setting of a clinical trial (Type: informal consensus, benefits/harms ratio uncertain; Evidence quality: insufficient; Strength of recommendation: weak).

\section{Recommendation 4.11. Radiographic progression on con- ventional imaging}

In men with metastatic CRPC with clear evidence of radiographic progression on conventional imaging while on systemic therapy, NGI should not be routinely offered. NGI may play a role if performed at baseline to facilitate comparison of imaging findings/extent of progression of disease (Type: consensus, benefits/harms ratio uncertain; Evidence quality: insufficient; Strength of recommendation: moderate).

\section{DISCUSSION}

There has been tremendous excitement in the prostate cancer community for advanced, molecular-based, NGI. This is driven in large part by the biology of the disease as well as by the clinical need to accurately stage patients and assess the burden and extent of disease. Prostate cancer categorization utilizes the clinical disease states model, which is determined by tumor characteristics, radiographic extent of disease, and prior therapies administered, making accurate imaging paramount. The anatomy of the prostate in the pelvis, with the very delicate surrounding structures that control crucial bodily functions (urinary, rectal, and sexual function), makes accurate assessment of extent of local disease crucial. For men whose disease is no longer localized and widespread, aggressive local therapy that will adversely impact urinary, sexual, and bowel domains could carry unacceptably high adverse effects with detriment to quality of life. Conversely, aggressive local therapy that could offer potential cure may be inappropriately withheld when conventional clinical parameters, such as PSA or Gleason score, deem a man high risk, for which NGI could indicate organ-confined disease without evidence of distant disease. The availability and clinical utility of PSA, an exquisitely sensitive serum-based tumor marker for prostate cancer, can add to the complexity of accurately determining the disease state, again highlighting the importance of accurate imaging. Following the disease states model, we have drafted our recommendations utilizing common clinical scenarios in the natural history of prostate cancer treatment, for which appropriate use of imaging can be categorized.

The primary driver for obtaining imaging should be when clinicians and patients are at a treatment nexus; therefore, imaging studies that will not impact or inform treatment decisions should be minimized. The urge or instinct to order multiple imaging modalities is common for solid tumor oncology, particularly to accurately assess burden of disease and risk to the patient. The overuse of imaging, especially NGI, does carry risks of increased cost, inappropriate ionizing radiation delivery to patients, and the risk of false-positive findings, which generate fear and anxiety for patients and clinicians as well as generate other unnecessary interventions.

As previously stated, the decision for NGI for patients experiencing biochemical recurrence is predicated on the potential treatment plan. If no additional salvage therapy, such as salvage radical prostatectomy, salvage radiotherapy, salvage ablative therapy (eg, cryotherapy, highintensity focused ultrasound therapy), or salvage lymphadenectomy, is planned because of patient concerns or preference due to potential adverse effects or medical comorbidities that preclude aggressive therapy, then the utilization and benefit of NGl is questionable.

In the arena of PET-directed imaging, there are multiple compounds in use that have been proposed, including small-molecule agents targeting PSMA as well as amino acid and fatty acid agents. There is a paucity of comparative data among these agents, and accurate comparisons between them are limited. This is an area in the future where prospective comparative studies would clarify the appropriate role and utility of these agents.

Similarly, there have been multiple isotopes in use with varying half-lives and source generators. These include primarily ${ }^{11} \mathrm{C},{ }^{18} \mathrm{~F}$, and ${ }^{68} \mathrm{Ga}$, with several smaller studies investigating other isotopes. Comparative studies to assess the accuracy of imaging different isotopes are also lacking, and the clinical implementation of specific isotopes have been driven mostly by half-life, ease of distribution, and method of isotope generation. This is another area of opportunity for analysis in the future.

In reviewing the published literature, there is a paucity of well-designed prospective studies in NGI for prostate cancer, and this Guideline Panel endeavored to create a reasonable, evidence-based, malleable framework to guide the optimal use of imaging in patients with advanced prostate cancer. As technology evolves and current and future prospective evaluations of NGI become available, we expect that these guidelines may require updates or changes in the future.

The Advanced Prostate Cancer Consensus Conference 2017 guideline ${ }^{99}$ recommended that imaging be performed at baseline, PSA nadir, and progression at least in patients with the usual presentation of metastatic hormone-sensitive prostate cancer. Imaging should also be performed in between these dates if there are additional clinical needs. Additionally, regular imaging monitoring of disease is recommended if there is a likelihood of aggressive variant prostate cancer (including small-cell and neuroendocrine) 
when the following clinical/pathologic/imaging features are present ${ }^{100}$ :

- Exclusively visceral metastases

- Radiographically predominant lytic bone metastases by plain x-ray or CT scan

- Bulky ( $\geq 5 \mathrm{~cm}$ ) lymphadenopathy or bulky ( $\geq 5 \mathrm{~cm}$ ) high-grade (Gleason $\geq 8$ ) tumor mass in prostate/ pelvis

- Low PSA ( $\leq 10 \mathrm{ng} / \mathrm{mL}$ ) at initial presentation (prior to ADT or at symptomatic progression in the castrate setting) plus high-volume ( $\geq 20)$ bone metastases

- Presence of neuroendocrine markers on histology or in serum; marked hypercalcemia

- Raised carcinoembryonic antigen

- Short interval ( $\leq 6$ months) to androgen-independent progression following the initiation of hormonal therapy with or without the presence of neuroendocrine markers

\section{SPECIAL COMMENTARY}

The recommendations provided are based on systematic literature review and do not specifically address the lack of universal availability of NGI modalities worldwide. Approval and payment for many NGI modalities have been hampered in the United States and other countries, both regionally and nationally, with significant angst for both patients and clinicians. This aspect is fluid and evolving, and availability should be considered when clinicians and patients pursue specific NGI modalities. As more evidence is presented that support the clinical utility of these imaging modalities, the availability of these tests may widen in the future.

\section{PATIENT AND CLINICIAN COMMUNICATION}

In panel discussions, there was robust discussion about patient counseling and concerns for false-positive and false-negative imaging results for conventional imaging as well as for NGI. It should be acknowledged that improved sensitivity for detection of low-volume metastatic disease may not be clinically relevant if there is a high false-positive rate, with attendant secondary testing, biopsy, and so forth. Additionally, there was also agreement that patients should be counseled on the life-long risk of ionizing radiation that NGI testing, particularly involving CT (eg, PET/CT), carry with the risk of subsequent malignancies. While perhaps not relevant for patients with advanced prostate cancer who have exhausted multiple lines of therapy, this does pertain to men in earlier disease states. Awareness of these issues with careful and deliberate communication is recommended between clinicians and patients.

For recommendations and strategies to optimize patientclinician communication, see Patient-Clinician Communication: American Society of Clinical Oncology Consensus Guideline. $^{101}$

\section{HEALTH DISPARITIES}

Although ASCO clinical practice guidelines represent expert recommendations on the best practices in disease management to provide the highest level of cancer care, it is important to note that many patients have limited access to medical care. Racial and ethnic disparities in health care contribute significantly to this problem in the United States. Patients with cancer who are members of racial/ethnic minorities suffer disproportionately from comorbidities, experience more substantial obstacles to receiving care, are more likely to be uninsured, and are at greater risk of receiving care of poor quality than other Americans. ${ }^{102-105}$ Many other patients lack access to care because of their geographic location and distance from appropriate treatment facilities. Awareness of these disparities in access to care should be considered in the context of this clinical practice guideline, and health care providers should strive to deliver the highest level of cancer care to these vulnerable populations.

\section{MULTIPLE CHRONIC CONDITIONS}

Creating evidence-based recommendations to inform treatment of patients with additional chronic conditions, a situation in which the patient may have two or more such conditions-referred to as multiple chronic conditions (MCCs) - is challenging. Patients with MCCs are a complex and heterogeneous population, making it difficult to account for all of the possible permutations to develop specific recommendations for care. In addition, the best available evidence for treating index conditions, such as cancer, is often from clinical trials whose study selection criteria may exclude these patients to avoid potential interaction effects or confounding of results associated with MCCs. As a result, the reliability of outcome data from these studies may be limited, thereby creating constraints for expert groups to make recommendations for care in this heterogeneous patient population.

As many patients for whom guideline recommendations apply present with MCCs, any treatment plan needs to take into account the complexity and uncertainty created by the presence of MCCs and highlights the importance of shared decision making regarding guideline use and implementation. Therefore, in consideration of recommended care for the target index condition, clinicians should review all other chronic conditions present in the patient and take those conditions into account when formulating the treatment and follow-up plan.

For patients with prostate cancer under 65 years of age, the 10 most common comorbidities are (in descending order) hypertension, hyperlipidemia, diabetes, ischemic heart disease, anemia, arthritis, chronic kidney disease, depression, chronic obstructive pulmonary disease (COPD), and heart failure. For patients with prostate cancer over 65 years of age, the 10 most common comorbidities are (in 
descending order) hypertension, hyperlipidemia, ischemic heart disease, anemia, diabetes, arthritis, chronic kidney disease, cataract, heart failure, and COPD.

In light of these considerations, practice guidelines should provide information on how to apply the recommendations for patients with MCCs, perhaps as a qualifying statement for recommended care. This may mean that some or all of the recommended care options are modified or not applied, as determined by best practice in consideration of any MCC.

Men with advanced prostate cancer commonly may have medical comorbidities and chronic kidney disease, which preclude the use of iodinated contrast or gadolinium. This may limit the applicability and administration of conventional and NGI in specific patient populations. An individualized approach is recommended to account for these conditions.

\section{COST IMPLICATIONS}

There has been a dramatic expansion in health care expenditures over the past 2 decades, and imaging technologies represent one of the fastest growing areas of health care spending. In particular, advanced imaging technologies, such as MRI, CT, and PET, may drive as much as $50 \%$ of these increased costs. ${ }^{106}$ Policy levers may be able to control some of these spiraling costs ${ }^{107}$; however, increasingly, individuals diagnosed with cancer are required to pay a larger proportion of their treatment costs. ${ }^{108-110}$ These higher out-of-pocket payments may represent a barrier to the initiation of and adherence to recommended cancer treatments. ${ }^{111-113}$ Therefore, a tailored discussion of potential diagnostic and treatment costs is an important component of shared treatment decision making. ${ }^{114}$ When feasible, clinicians should counsel patients regarding the use of less expensive alternatives for diagnosis and treatment when there is clinical equipoise or rapidly evolving therapy options, such as the case with imaging for advanced prostate cancer. ${ }^{114}$

Importantly, there is considerable heterogeneity in health care costs for diagnostic imaging. Variation in expenditure may be related to region, payer, hospital system, negotiated contracts, insurance status, or severity of illness, and this variability precludes a systematic and generalizable evaluation. ${ }^{115}$ With regard to advanced imaging modalities in particular, there is substantial variation in organizational costs related to isotope procurement from external sources ( ${ }^{18} \mathrm{~F}$-choline), possible cyclotron expenses ( ${ }^{11} \mathrm{C}$-choline), generator expenses, and radiopharmacy ( ${ }^{68} \mathrm{Ga}$-PSMA) as well as the direct costs of disposable equipment and indirect costs of physicians, technologists, and radiopharmacists. ${ }^{116}$ When discussing financial issues surrounding care delivery, patients should be made aware of any economic counseling services available to address this very complex landscape. ${ }^{114}$
Given the recent and rapid expansion of imaging modalities for advanced prostate cancer, there are no exhaustive comparative effectiveness analyses that encompass each of the techniques described in this guideline. There are data that suggest that advanced imaging modalities can be cost effective for patients with cancer, in general, particularly when used for monitoring therapy, staging, or diagnosis, rather than screening. ${ }^{117} \mathrm{~A}$ formal costeffectiveness evaluation of advanced imaging for patients with prostate cancer will have to balance the important patient-level factors specifically associated with a prostate cancer diagnosis, such as the relative longevity associated with a high-risk prostate cancer diagnosis, the loss of physical productivity, and the substantial costs associated with skeletal-related events. ${ }^{118-120}$

As this field continues to rapidly expand and we endeavor to clarify the most sensitive and cost-effective imaging modality for advanced prostate cancer, it will be important to consider the potential impact of the inevitable regionalization and the subsequent access challenges that these emerging, advanced technologies will likely create. This will be a particularly important consideration for populations at already increased risk of health care disparities.

\section{EXTERNAL REVIEW AND OPEN COMMENT}

The draft recommendations were released to the public for open comment from February 22, 2019, through March 8, 2019. Response categories of "Agree as written," "Agree with suggested modifications," and "Disagree. See comments" were captured for every proposed recommendation, with 13 submissions received. A total of $100 \%$ of the 13 respondents either agreed or agreed with slight modifications to the recommendations, and $0 \%$ of the respondents disagreed. Expert Panel members reviewed comments from all sources and determined whether to maintain original draft recommendations, revise with minor language changes, or consider major recommendation revisions. All changes were incorporated prior to CPGC review and approval.

The draft was submitted to 2 external reviewers with content expertise. It was rated as high quality, and it was agreed that it would be useful in practice. Comments received assisted in presenting the strength of the evidence that supported each of the recommendations and helped to contextualize this guideline in relation to other ASCO guidelines within this disease site. Review comments were reviewed by the Expert Panel and integrated into the final manuscript before approval by the CPGC.

\section{GUIDELINE IMPLEMENTATION}

ASCO guidelines are developed for implementation across health settings. Barriers to implementation include the need to increase awareness of the guideline 
recommendations among frontline practitioners and survivors of cancer and caregivers as well as to provide adequate services in the face of limited resources. The guideline Bottom Line Box was designed to facilitate implementation of recommendations. This guideline will be distributed widely through the ASCO Practice Guideline Implementation Network. ASCO guidelines are posted on the ASCO web site and most often published in $\mathrm{JCO}$ and a summary in Journal of Oncology Practice.

\section{LIMITATIONS OF THE RESEARCH AND FUTURE RESEARCH}

This guideline is based on the best available evidence regarding the use of imaging in advanced prostate cancer. However, it is recognized that there are gaps in knowledge related to insufficiency or absence of data in various scenarios related to this condition. Specifically, there are limited data on head-to-head comparisons of diagnostic performance of different imaging modalities or different radiopharmaceutical agents in the same patient population. Also, while there is evidence of feasibility of use and change in management as a result of imaging findings, none of the imaging modalities show prospective evidence conferring patient benefit in terms of outcomes (ie, how patients feel, function, or survive). These should be areas for focused future research. Finally, some of the agents that have been shown to have higher detection capabilities in prostate cancer (eg,

\section{AFFILIATIONS}

${ }^{1}$ Sidney Kimmel Medical College at Thomas Jefferson University, Philadelphia, PA

${ }^{2}$ American Society of Clinical Oncology, Alexandria, VA

${ }^{3}$ University of Southern California, Los Angeles, CA

${ }^{4}$ University of California, San Francisco, San Francisco, CA

${ }^{5}$ Johns Hopkins Medicine, Owings Mills, MD

${ }^{6}$ National Cancer Institute, Bethesda, MD

${ }^{7} \mathrm{NYU}$ Langone Health, New York, NY

${ }^{8}$ University of Cincinnati Medical Center, Cincinnati, $\mathrm{OH}$

${ }^{9}$ David Geffen School of Medicine, Los Angeles, CA

${ }^{10}$ Mayo Clinic, Rochester, MN

${ }^{11}$ The University of Chicago, Chicago, IL

${ }^{12} \mathrm{Cleveland}$ Clinic, Cleveland, $\mathrm{OH}$

${ }^{13}$ UNC Lineberger Comprehensive Cancer Center, Chapel Hill, NC

${ }^{14}$ German Cancer Research Center (DKFZ), Heidelberg, Germany

${ }^{15}$ Technische Universität München, Munich, Germany

${ }^{16}$ Memorial Sloan Kettering Cancer Center, New York, NY

${ }^{17}$ Paul Strickland Scanner Centre, Mount Vernon Cancer Centre, Northwood, United Kingdom

${ }^{18}$ University of Bologna, Bologna, Italy

${ }^{19}$ Queen's University Belfast, Belfast, Northern Ireland

${ }^{20}$ Vanderbilt Urologic Surgery, Nashville, TN

${ }^{21}$ Virginia Mason Medical Center, Seattle, WA

${ }^{22}$ London Health Sciences Centre, London, Ontario, Canada

${ }^{23}$ Winship Cancer Institute, Atlanta, GA

${ }^{24}$ Hartford Hospital, Hartford, CT

\section{CORRESPONDING AUTHOR}

American Society of Clinical Oncology, 2318 Mill Rd, Suite 800, Alexandria, VA 22314; e-mail: guidelines@asco.org.
PSMA) do not have regulatory approval for use in the United States.

\section{ADDITIONAL RESOURCES}

More information, including a Data Supplement with additional evidence tables, slide sets, and clinical tools and resources, is available at www.asco.org/genitourinarycancer-guidelines. Patient information is available at www.cancer.net.

\section{RELATED ASCO GUIDELINES}

- Integration of Palliative Care Into Standard Oncology Practice ${ }^{121} \quad$ (http://ascopubs.org/doi/ 10.1200/JC0.2016.70.1474)

- Patient-Clinician Communication ${ }^{101}$ (http:// ascopubs.org/doi/10.1200/JC0.2017.75.2311)

- Hypofractionated Radiation Therapy for Localized Prostate Cancer ${ }^{122}$ (http://ascopubs.org/doi/ 10.1200/JC0.18.01097)

- Clinically Localized Prostate Cancer ${ }^{123}$ (http:// ascopubs.org/doi/10.1200/JC0.18.00606)

- Optimizing Anticancer Therapy in Metastatic Noncastrate Prostate Cancer ${ }^{124}$ (http:// ascopubs.org/doi/10.1200/JC0.2018.78.0619)

\section{EDITOR'S NOTE}

This American Society of Clinical Oncology (ASCO) Clinical Practice Guideline provides recommendations, with comprehensive review and analyses of the relevant literature for each recommendation. Additional information, including a Data Supplement with additional evidence tables, slide sets, clinical tools and resources, and links to patient information at www.cancer.net, is available at www.asco.org/ genitourinary-cancer-guidelines.

\section{EQUAL CONTRIBUTION}

E.J.T. and H.A.V. were Expert Panel co-chairs.

Clinical Practice Guideline Committee approval: October 4, 2019 Reprint Requests: 2318 Mill Rd, Suite 800, Alexandria, VA 22314; guidelines@asco.org.

\section{AUTHORS' DISCLOSURES OF POTENTIAL CONFLICTS OF INTEREST AND DATA AVAILABILITY STATEMENT}

Disclosures provided by the authors and data availability statement (if applicable) are available with this article at DOI https://doi.org/10.1200/ JCO.19.02757. 


\section{AUTHOR CONTRIBUTIONS}

Conception and design: All authors

Collection and assembly of data: All authors

Data analysis and interpretation: All authors

Manuscript writing: All authors

Final approval of manuscript: All authors

Accountable for all aspects of the work: All authors

\section{ACKNOWLEDGMENT}

The Expert Panel wishes to thank J. Kellogg Parsons, MD (CPGC reviewer), Peter J. Van Veldhuizen, MD (CPGC reviewer), Eric A. Singer, MD (external reviewer), Daniel E. Spratt, MD (external reviewer), Erin B. Kennedy, MHSc (internal reviewer), Thomas K. Oliver (internal reviewer), and Shannon E. McKernin (internal reviewer), and the Clinical Practice Guidelines Committee for their thoughtful reviews and insightful comments on this guideline.

\section{REFERENCES}

1. Sanda MG, Cadeddu JA, Kirkby E, et al: Clinically localized prostate cancer: AUA/ASTRO/SUO guideline. Part I: Risk stratification, shared decision making, and care options. J Urol 199:683-690, 2018

2. Siegel RL, Miller KD, Jemal A: Cancer statistics, 2019. CA Cancer J Clin 69:7-34, 2019

3. Ryan CJ, Shah S, Efstathiou E, et al: Phase II study of abiraterone acetate plus prednisone in chemotherapy-naïve metastatic castration-resistant prostate cancer demonstrating radiographic flare discordant with serologic measures of response. Clin Cancer Res 17:4854-4861, 2011

4. Scher HI, Halabi S, Tannock I, et al: Design and end points of clinical trials for patients with progressive prostate cancer and castrate levels of testosterone: Recommendations of the Prostate Cancer Clinical Trials Working Group. J Clin Oncol 26:1148-1159, 2008

5. Scher HI, Morris MJ, Stadler WM, et al: Trial design and objectives for castration-resistant prostate cancer: Updated recommendations from the Prostate Cancer Clinical Trials Working Group 3. J Clin Oncol 34:1402-1418, 2016

6. Kluetz PG, Pierce W, Maher VE, et al: Radium Ra 223 dichloride injection: U.S. Food and Drug Administration drug approval summary. Clin Cancer Res 20:914,2013

7. Morris MJ, Molina A, Small EJ, et al: Radiographic progression-free survival as a response biomarker in metastatic castration-resistant prostate cancer: COUAA-302 results. J Clin Oncol 33:1356-1363, 2015

8. Rathkopf DE, Beer TM, Loriot Y, et al: Radiographic progression-free survival as a clinically meaningful end point in metastatic castration-resistant prostate cancer: The PREVAIL randomized clinical trial. JAMA Oncol 4:694-701, 2018

9. Imbriaco M, Larson SM, Yeung HW, et al: A new parameter for measuring metastatic bone involvement by prostate cancer: The Bone Scan Index. Clin Cancer Res 4:1765-1772, 1998

10. Anand A, Morris MJ, Kaboteh R, et al: Analytic validation of the automated bone scan index as an imaging biomarker to standardize quantitative changes in bone scans of patients with metastatic prostate cancer. J Nucl Med 57:41-45, 2016

11. Armstrong AJ, Anand A, Edenbrandt L, et al: Phase 3 assessment of the automated bone scan index as a prognostic imaging biomarker of overall survival in men with metastatic castration-resistant prostate cancer: A secondary analysis of a randomized clinical trial. JAMA Oncol 4:944-951, 2018

12. Bach-Gansmo T, Nanni C, Nieh PT, et al: Multisite experience of the safety, detection rate and diagnostic performance of fluciclovine ( ${ }^{18} \mathrm{~F}$ ) positron emission tomography/computerized tomography imaging in the staging of biochemically recurrent prostate cancer. J Urol 197:676-683, 2017

13. Vapiwala N, Hofman MS, Murphy DG, et al: Strategies for evaluation of novel imaging in prostate cancer: Putting the horse back before the cart. J Clin Oncol 37:765-769, 2019

14. Christensen D: The Will Rogers phenomenon: Roping the effects of a new cancer staging system. J Natl Cancer Inst 95:1105-1106, 2003

15. Roach M III, Hanks G, Thames H Jr, et al: Defining biochemical failure following radiotherapy with or without hormonal therapy in men with clinically localized prostate cancer: Recommendations of the RTOG-ASTRO Phoenix Consensus Conference. Int J Radiat Oncol Biol Phys 65:965-974, 2006

16. Tilki D, Kim SI, Hu B, et al: Ultrasensitive prostate specific antigen and its role after radical prostatectomy: A systematic review. J Urol 193:1525-1531, 2015

17. Shiffman RN, Michel G, Rosenfeld RM, et al: Building better guidelines with BRIDGE-Wiz: Development and evaluation of a software assistant to promote clarity, transparency, and implementability. J Am Med Inform Assoc 19:94-101, 2012

18. Fitzpatrick C, Lynch O, Marignol L: ${ }^{68} \mathrm{Ga}-\mathrm{PSMA}-\mathrm{PET} / \mathrm{CT}$ has a role in detecting prostate cancer lesions in patients with recurrent disease. Anticancer Res 37 : 2753-2760, 2017

19. von Eyben FE, Kairemo K: Acquisition with (11)C-choline and (18)F-fluorocholine PET/CT for patients with biochemical recurrence of prostate cancer: A systematic review and meta-analysis. Ann Nucl Med 30:385-392, 2016

20. von Eyben FE, Picchio M, von Eyben R, et al: 68Ga-labeled prostate-specific membrane antigen ligand positron emission tomography/computed tomography for prostate cancer: A systematic review and meta-analysis. Eur Urol Focus 4:686-693, 2018

21. Ren J, Yuan L, Wen G, et al: The value of anti-1-amino-3- ${ }^{18}$ F-fluorocyclobutane-1-carboxylic acid PET/CT in the diagnosis of recurrent prostate carcinoma: A meta-analysis. Acta Radiol 57:487-493, 2016

22. Perera M, Papa N, Christidis D, et al: Sensitivity, specificity, and predictors of positive ${ }^{68}$ Ga-prostate-specific membrane antigen positron emission tomography in advanced prostate cancer: A systematic review and meta-analysis. Eur Urol 70:926-937, 2016

23. Liu J, Chen Z, Wang T, et al: Influence of four radiotracers in PET/CT on diagnostic accuracy for prostate cancer: A bivariate random-effects meta-analysis. Cell Physiol Biochem 39:467-480, 2016

24. Fanti S, Minozzi S, Castellucci P, et al: PET/CT with (11)C-choline for evaluation of prostate cancer patients with biochemical recurrence: Meta-analysis and critical review of available data. Eur J Nucl Med Mol Imaging 43:55-69, 2016

25. de Rooij M, Hamoen EH, Witjes JA, et al: Accuracy of magnetic resonance imaging for local staging of prostate cancer: A diagnostic meta-analysis. Eur Urol 70: 233-245, 2016

26. von Eyben FE, Kairemo K: Meta-analysis of (11)C-choline and (18)F-choline PET/CT for management of patients with prostate cancer. Nucl Med Commun 35: 221-230, 2014

27. Shen G, Deng H, Hu S, et al: Comparison of choline-PET/CT, MRI, SPECT, and bone scintigraphy in the diagnosis of bone metastases in patients with prostate cancer: A meta-analysis. Skeletal Radiol 43:1503-1513, 2014

28. Treglia G, Ceriani L, Sadeghi R, et al: Relationship between prostate-specific antigen kinetics and detection rate of radiolabelled choline PET/CT in restaging prostate cancer patients: A meta-analysis. Clin Chem Lab Med 52:725-733, 2014

29. Umbehr MH, Müntener M, Hany T, et al: The role of ${ }^{11} \mathrm{C}$-choline and ${ }^{18} \mathrm{~F}$-fluorocholine positron emission tomography (PET) and PET/CT in prostate cancer: $\mathrm{A}$ systematic review and meta-analysis. Eur Urol 64:106-117, 2013 
30. Mohsen B, Giorgio T, Rasoul ZS, et al: Application of C-11-acetate positron-emission tomography (PET) imaging in prostate cancer: Systematic review and meta-analysis of the literature. BJU Int 112:1062-1072, 2013

31. Silva RC, Sasse AD, Matheus WE, et al: Magnetic resonance image in the diagnosis and evaluation of extra-prostatic extension and involvement of seminal vesicles of prostate cancer: A systematic review of literature and meta-analysis. Int Braz J Urol 39:155-166, 2013

32. Evangelista L, Zattoni F, Guttilla A, et al: Choline PET or PET/CT and biochemical relapse of prostate cancer: A systematic review and meta-analysis. Clin Nucl Med 38:305-314, 2013

33. Evangelista L, Guttilla A, Zattoni F, et al: Utility of choline positron emission tomography/computed tomography for lymph node involvement identification in intermediate- to high-risk prostate cancer: A systematic literature review and meta-analysis. Eur Urol 63:1040-1048, 2013

34. Alfarone A, Panebianco V, Schillaci $\mathrm{O}$, et al: Comparative analysis of multiparametric magnetic resonance and PET-CT in the management of local recurrence after radical prostatectomy for prostate cancer. Crit Rev Oncol Hematol 84:109-121, 2012

35. Schmidkonz C, Cordes M, Beck M, et al: SPECT/CT With the PSMA ligand 99m-Tc-MIP-1404 for whole-body primary staging of patients with prostate cancer. Clin Nucl Med 43:225-231, 2018

36. Schmidkonz C, Hollweg C, Beck M, et al: 99m TC-MIP-1404-SPECT/CT for the detection of PSMA-positive lesions in 225 patients with biochemical recurrence of prostate cancer. Prostate 78:54-63, 2018

37. Habl G, Sauter K, Schiller K, et al: ${ }^{68}$ Ga-PSMA-PET for radiation treatment planning in prostate cancer recurrences after surgery: Individualized medicine or new standard in salvage treatment. Prostate 77:920-927, 2017

38. Goffin KE, Joniau S, Tenke P, et al: Phase 2 study of ${ }^{99 m}$ TC-Trofolastat SPECT/CT to identify and localize prostate cancer in intermediate- and high-risk patients undergoing radical prostatectomy and extended pelvic LN dissection. J Nucl Med 58:1408-1413, 2017

39. Freitag MT, Radtke JP, Afshar-Oromieh A, et al: Local recurrence of prostate cancer after radical prostatectomy is at risk to be missed in ${ }^{68} \mathrm{Ga}-\mathrm{PSMA}-11-\mathrm{PET}$ of PET/CT and PET/MRI: Comparison with mpMRI integrated in simultaneous PET/MRI. Eur J Nucl Med Mol Imaging 44:776-787, 2017

40. Einspieler I, Rauscher I, Düwel C, et al: Detection efficacy of hybrid ${ }^{68} \mathrm{Ga}-\mathrm{PSMA}$ ligand PET/CT in prostate cancer patients with biochemical recurrence after primary radiation therapy defined by Phoenix criteria. J Nucl Med 58:1081-1087, 2017

41. Dietlein F, Kobe C, Neubauer S, et al: PSA-stratified performance of ${ }^{18} \mathrm{~F}$ - and ${ }^{68} \mathrm{Ga}$-PSMA PET in patients with biochemical recurrence of prostate cancer. J Nucl Med 58:947-952, 2017

42. Berliner C, Tienken M, Frenzel T, et al: Detection rate of PET/CT in patients with biochemical relapse of prostate cancer using [ $\left.{ }^{68} \mathrm{Ga}\right] \mathrm{PSMA}$ I\&T and comparison with published data of [ ${ }^{68} \mathrm{Ga}$ ]PSMA HBED-CC. Eur J Nucl Med Mol Imaging 44:670-677, 2017

43. Albisinni S, Artigas C, Aoun F, et al: Clinical impact of ${ }^{68}$ Ga-prostate-specific membrane antigen (PSMA) positron emission tomography/computed tomography (PET/CT) in patients with prostate cancer with rising prostate-specific antigen after treatment with curative intent: Preliminary analysis of a multidisciplinary approach. BJU Int 120:197-203, 2017

44. Akin-Akintayo 00 , Jani $\mathrm{AB}$, Odewole $\mathrm{O}$, et al: Change in salvage radiotherapy management based on guidance with FACBC (Fluciclovine) PET/CT in postprostatectomy recurrent prostate cancer. Clin Nucl Med 42:e22-e28, 2017

45. Rahbar K, Schmidt M, Heinzel A, et al: Response and tolerability of a single dose of ${ }^{177}$ Lu-PSMA-617 in patients with metastatic castration-resistant prostate cancer: A multicenter retrospective analysis. J Nucl Med 57:1334-1338, 2016

46. Pfister D, Porres D, Heidenreich A, et al: Detection of recurrent prostate cancer lesions before salvage lymphadenectomy is more accurate with (68)Ga-PSMAHBED-CC than with (18)F-fluoroethylcholine PET/CT. Eur J Nucl Med Mol Imaging 43:1410-1417, 2016

47. Odewole OA, Tade FI, Nieh PT, et al: Recurrent prostate cancer detection with anti-3-[(18)F]FACBC PET/CT: Comparison with CT. Eur J Nucl Med Mol Imaging 43:1773-1783, 2016

48. Nanni C, Zanoni L, Pultrone C, et al: (18)F-FACBC (anti1-amino-3-(18)F-fluorocyclobutane-1-carboxylic acid) versus (11)C-choline PET/CT in prostate cancer relapse: Results of a prospective trial. Eur J Nucl Med Mol Imaging 43:1601-1610, 2016

49. Larbi A, Dallaudière B, Pasoglou V, et al: Whole body MRI (WB-MRI) assessment of metastatic spread in prostate cancer: Therapeutic perspectives on targeted management of oligometastatic disease. Prostate 76:1024-1033, 2016

50. Barchetti F, Stagnitti A, Megna V, et al: Unenhanced whole-body MRI versus PET-CT for the detection of prostate cancer metastases after primary treatment. Eur Rev Med Pharmacol Sci 20:3770-3776, 2016

51. Ceci F, Uprimny C, Nilica B, et al: (68)Ga-PSMA PET/CT for restaging recurrent prostate cancer: Which factors are associated with PET/CT detection rate? Eur J Nucl Med Mol Imaging 42:1284-1294, 2015

52. Schuster DM, Nieh PT, Jani AB, et al: Anti-3-[(18)F]FACBC positron emission tomography-computerized tomography and (111)In-capromab pendetide single photon emission computerized tomography-computerized tomography for recurrent prostate carcinoma: Results of a prospective clinical trial. J Urol 191:1446-1453, 2014

53. Harisinghani MG, Barentsz J, Hahn PF, et al: Noninvasive detection of clinically occult lymph-node metastases in prostate cancer. N Engl J Med 348: 2491-2499, 2003

54. Evangelista L, Cimitan M, Zattoni F, et al: Comparison between conventional imaging (abdominal-pelvic computed tomography and bone scan) and [(18)F] choline positron emission tomography/computed tomography imaging for the initial staging of patients with intermediate- to high-risk prostate cancer: A retrospective analysis. Scand J Urol 49:345-353, 2015

55. Parker SJ, Pond GR, Agarwal N, et al: Integration of bone and computed tomography scans to assess bone metastasis in metastatic castration-resistant prostate cancer. Clin Genitourin Cancer 15:53-59, 2017

56. Tombal B, Rezazadeh A, Therasse P, et al: Magnetic resonance imaging of the axial skeleton enables objective measurement of tumor response on prostate cancer bone metastases. Prostate 65:178-187, 2005

57. Levenson RM, Sauerbrunn BJ, Bates HR, et al: Comparative value of bone scintigraphy and radiography in monitoring tumor response in systemically treated prostatic carcinoma. Radiology 146:513-518, 1983

58. Pollen JJ, Witztum KF, Ashburn WL: The flare phenomenon on radionuclide bone scan in metastatic prostate cancer. AJR Am J Roentgenol 142:773-776, 1984

59. Li D, Lv H, Hao X, et al: Prognostic value of bone scan index as an imaging biomarker in metastatic prostate cancer: A meta-analysis. Oncotarget 8: 84449-84458, 2017

60. Suh $\mathrm{CH}$, Shinagare $\mathrm{AB}$, Westenfield $\mathrm{AM}$, et al: Yield of bone scintigraphy for the detection of metastatic disease in treatment-naive prostate cancer: $\mathrm{A}$ systematic review and meta-analysis. Clin Radiol 73:158-167, 2018

61. Jadvar H: Imaging evaluation of prostate cancer with ${ }^{18} \mathrm{~F}$-fluorodeoxyglucose PET/CT: Utility and limitations. Eur J Nucl Med Mol Imaging 40:S5-S10, 2013

62. Kaittanis $\mathrm{C}$, Andreou C, Hieronymus $\mathrm{H}$, et al: Prostate-specific membrane antigen cleavage of vitamin B9 stimulates oncogenic signaling through metabotropic glutamate receptors. J Exp Med 215:159-175, 2018 [Erratum: J Exp Med 215:377, 2018] 
63. Hope TA, Afshar-Oromieh A, Eiber M, et al: Imaging prostate cancer with prostate-specific membrane antigen PET/CT and PET/MRI: Current and future applications. AJR Am J Roentgenol 211:286-294, 2018

64. Rahbar K, Afshar-Oromieh A, Jadvar H, et al: PSMA theranostics: Current status and future directions. Mol Imaging 17:1536012118776068, 2018

65. Hofman MS, Hicks RJ, Maurer T, et al: Prostate-specific membrane antigen PET: Clinical utility in prostate cancer, normal patterns, pearls, and pitfalls. Radiographics 38:200-217, 2018

66. Eiber M, Herrmann K, Calais J, et al: Prostate cancer molecular imaging standardized evaluation (PROMISE): Proposed miTNM classification for the interpretation of PSMA-ligand PET/CT. J Nucl Med 59:469-478, 2018

67. Fendler WP, Eiber M, Beheshti M, et al: ${ }^{68} \mathrm{Ga}-\mathrm{PSMA}$ PET/CT: Joint EANM and SNMMI procedure guideline for prostate cancer imaging: Version 1.0. Eur J Nucl Med Mol Imaging 44:1014-1024, 2017

68. Rauscher I, Maurer T, Fendler WP, et al: (68)Ga-PSMA ligand PET/CT in patients with prostate cancer: How we review and report. Cancer Imaging 16:14, 2016

69. Rowe SP, Pienta KJ, Pomper MG, et al: Proposal for a structured reporting system for prostate-specific membrane antigen-targeted PET imaging: PSMA-RADS version 1.0. J Nucl Med 59:479-485, 2018

70. Sheikhbahaei S, Afshar-Oromieh A, Eiber M, et al: Pearls and pitfalls in clinical interpretation of prostate-specific membrane antigen (PSMA)-targeted PET imaging. Eur J Nucl Med Mol Imaging 44:2117-2136, 2017

71. Calais J, Czernin J, Cao M, et al: ${ }^{68} \mathrm{Ga}-\mathrm{PSMA}-11 \mathrm{PET} / \mathrm{CT}$ mapping of prostate cancer biochemical recurrence after radical prostatectomy in 270 patients with a PSA level of less than $1.0 \mathrm{ng} / \mathrm{mL}$ : Impact on salvage radiotherapy planning. J Nucl Med 59:230-237, 2018

72. Hope TA, Aggarwal R, Chee B, et al: Impact of ${ }^{68}$ Ga-PSMA-11 PET on management in patients with biochemically recurrent prostate cancer. J Nucl Med 58: 1956-1961, 2017

73. Roach PJ, Francis R, Emmett L, et al: The impact of ${ }^{68} \mathrm{Ga}-\mathrm{PSMA}$ PET/CT on management intent in prostate cancer: Results of an Australian prospective multicenter study. J Nucl Med 59:82-88, 2018

74. Sathianathen NJ, Butaney M, Konety BR: The utility of PET-based imaging for prostate cancer biochemical recurrence: A systematic review and metaanalysis. World J Urol 37:1239-1249, 2019

75. Evans JD, Jethwa KR, Ost P, et al: Prostate cancer-specific PET radiotracers: A review on the clinical utility in recurrent disease. Pract Radiat Oncol 8:28-39, 2018

76. Lindenberg L, Ahlman M, Turkbey B, et al: Advancement of MR and PET/MR in prostate cancer. Semin Nucl Med 46:536-543, 2016

77. Afshar-Oromieh A, Zechmann CM, Malcher A, et al: Comparison of PET imaging with a (68)Ga-labelled PSMA ligand and (18)F-choline-based PET/CT for the diagnosis of recurrent prostate cancer. Eur J Nucl Med Mol Imaging 41:11-20, 2014

78. Al-Bayati M, Grueneisen J, Lütje S, et al: Integrated 68gallium labelled prostate-specific membrane antigen-11 positron emission tomography/magnetic resonance imaging enhances discriminatory power of multi-parametric prostate magnetic resonance imaging. Urol Int 100:164-171, 2018

79. Alonso O, Dos Santos G, García Fontes M, et al: ${ }^{68}$ Ga-PSMA and ${ }^{11} \mathrm{C}$-Choline comparison using a tri-modality PET/CT-MRI (3.0 T) system with a dedicated shuttle. Eur J Hybrid Imaging 2:9, 2018

80. Eiber M, Weirich G, Holzapfel K, et al: Simultaneous ${ }^{68}$ Ga-PSMA HBED-CC PET/MRI improves the localization of primary prostate cancer. Eur Urol 70: 829-836, 2016

81. Taneja S, Jena A, Taneja R, et al: Effect of combined ${ }^{68}$ Ga-PSMAHBED-CC uptake pattern and multiparametric MRI derived with simultaneous PET/MRI in the diagnosis of primary prostate cancer: Initial experience. AJR Am J Roentgenol 210:1338-1345, 2018

82. Elschot M, Selnæs KM, Sandsmark E, et al: Combined ${ }^{18} \mathrm{~F}$-fluciclovine PET/MRI shows potential for detection and characterization of high-risk prostate cancer. J Nucl Med 59:762-768, 2018

83. Elschot M, Selnæs KM, Sandsmark E, et al: A PET/MRI study towards finding the optimal $\left[{ }^{18} \mathrm{~F}\right]$ fluciclovine PET protocol for detection and characterisation of primary prostate cancer. Eur J Nucl Med Mol Imaging 44:695-703, 2017

84. Jambor I, Kuisma A, Ramadan S, et al: Prospective evaluation of planar bone scintigraphy, SPECT, SPECT/CT, ${ }^{18} \mathrm{~F}-\mathrm{NaF}$ PET/CT and whole body $1.5 \mathrm{~T}$ MRI, including DWI, for the detection of bone metastases in high risk breast and prostate cancer patients: SKELETA clinical trial. Acta Oncol 55:59-67, 2016

85. Lindenberg L, AhIman M, Turkbey B, et al: Evaluation of prostate cancer with PET/MRI. J Nucl Med 57:111S-116S, 2016

86. Grubmüller B, Baltzer P, Hartenbach S, et al: PSMA ligand PET/MRI for primary prostate cancer: Staging performance and clinical impact. Clin Cancer Res 24 : 6300-6307, 2018

87. Maurer T, Gschwend JE, Rauscher I, et al: Diagnostic efficacy of (68)gallium-PSMA positron emission tomography compared to conventional imaging for lymph node staging of 130 consecutive patients with intermediate to high risk prostate cancer. J Urol 195:1436-1443, 2016

88. Weber WA: PET/MR imaging: A critical appraisal. J Nucl Med 55:56S-58S, 2014

89. Schuster DM, Savir-Baruch B, Nieh PT, et al: Detection of recurrent prostate carcinoma with anti-1-amino-3-18 F-fluorocyclobutane-1-carboxylic acid PET/CT and ${ }^{111}$ In-capromab pendetide SPECT/CT. Radiology 259:852-861, 2011

90. Seltzer MA, Barbaric Z, Belldegrun A, et al: Comparison of helical computerized tomography, positron emission tomography and monoclonal antibody scans for evaluation of lymph node metastases in patients with prostate specific antigen relapse after treatment for localized prostate cancer. J Urol 162:1322-1328, 1999

91. Heidenreich A, OhImann CH, Polyakov S: Anatomical extent of pelvic lymphadenectomy in patients undergoing radical prostatectomy. Eur Urol 52:29-37, 2007

92. Padhani AR, Lecouvet FE, Tunariu N, et al: Rationale for modernising imaging in advanced prostate cancer. Eur Urol Focus 3:223-239, 2017

93. Liu LP, Cui LB, Zhang XX, et al: Diagnostic performance of diffusion-weighted magnetic resonance imaging in bone malignancy: Evidence from a metaanalysis. Medicine (Baltimore) 94:e1998, 2015

94. Lecouvet FE, El Mouedden J, Collette L, et al: Can whole-body magnetic resonance imaging with diffusion-weighted imaging replace Tc 99m bone scanning and computed tomography for single-step detection of metastases in patients with high-risk prostate cancer? Eur Urol 62:68-75, 2012

95. Dyrberg E, Hendel HW, Huynh THV, et al: 68Ga-PSMA-PET/CT in comparison with 18F-fluoride-PET/CT and whole-body MRI for the detection of bone metastases in patients with prostate cancer: A prospective diagnostic accuracy study. Eur Radiol 29:1221-1230, 2018

96. Hicks RM, Simko JP, Westphalen AC, et al: Diagnostic accuracy of ${ }^{68} \mathrm{Ga}-\mathrm{PSMA}-11$ PET/MRI compared with multiparametric MRI in the detection of prostate cancer. Radiology 289:730-737, 2018

97. Lengana T, Lawal IO, Boshomane TG, et al: ${ }^{68} \mathrm{Ga}-\mathrm{PSMA}$ PET/CT replacing bone scan in the initial staging of skeletal metastasis in prostate cancer: A fait accompli? Clin Genitourin Cancer 16:392-401, 2018

98. Scher HI, Morris MJ, Stadler WM, et al: (PCWG3) consensus for trials in castration-resistant prostate cancer (CRPC). J Clin Oncol 33, 2015 (suppl; abstr 5000) 
99. Gillessen S, Attard G, Beer TM, et al: Management of patients with advanced prostate cancer: The report of the Advanced Prostate Cancer Consensus Conference APCCC 2017. Eur Urol 73:178-211, 2018

100. Aparicio AM, Harzstark AL, Corn PG, et al: Platinum-based chemotherapy for variant castrate-resistant prostate cancer. Clin Cancer Res 19:3621-3630, 2013

101. Gilligan T, Coyle N, Frankel RM, et al: Patient-clinician communication: American Society of Clinical Oncology consensus guideline. J Clin Oncol 35: 3618-3632, 2017

102. American Cancer Society: Cancer Facts \& Figures for African Americans 2016-2018. Atlanta, GA, American Cancer Society, 2016

103. US Cancer Statistics Working Group: United States Cancer Statistics: 1999-2012 Incidence and Mortality Data. Atlanta, GA: US Department of Health and Human Services, Centers for Disease Control and Prevention and National Cancer Institute, 2015. www.cdc.gov/uscs

104. Howlader N, Noone AM, Krapcho M, et al: SEER Cancer Statistics Review, 1975-2013. http://seer.cancer.gov/csr/1975_2013

105. Mead H, Cartwright-Smith L, Jones K, et al: Racial and Ethnic Disparities in U.S. Health Care: A Chartbook. Washington, DC, The Commonwealth Fund, 2008

106. Loggers ET, Fishman PA, Peterson D, et al: Advanced imaging among health maintenance organization enrollees with cancer. J Oncol Pract 10:231-238, 2014

107. Noveiry BB, Varzaneh FN, Yousem DM: Radiologist revenue change following multiple-procedure payment reduction modification. J Am Coll Radiol 15: 941-942, 2018

108. Schnipper LE, Davidson NE, Wollins DS, et al: Updating the American Society of Clinical Oncology Value Framework: Revisions and reflections in response to comments received. J Clin Oncol 34:2925-2934, 2016

109. Schnipper LE, Davidson NE, Wollins DS, et al: American Society of Clinical Oncology statement: A conceptual framework to assess the value of cancer treatment options. J Clin Oncol 33:2563-2577, 2015

110. Lee DW, Levy F: The sharp slowdown in growth of medical imaging: An early analysis suggests combination of policies was the cause. Health Aff (Millwood) 31 : 1876-1884, 2012

111. Dusetzina SB, Winn AN, Abel GA, et al: Cost sharing and adherence to tyrosine kinase inhibitors for patients with chronic myeloid leukemia. J Clin Oncol 32 : 306-311, 2014

112. Streeter SB, Schwartzberg L, Husain N, et al: Patient and plan characteristics affecting abandonment of oral oncolytic prescriptions. J Oncol Pract 7:46s-51s, 2011

113. Rosenkrantz AB, Sadigh G, Carlos RC, et al: Out-of-pocket costs for advanced imaging across the US private insurance marketplace. J Am Coll Radiol 15:607614.e1, 2018

114. Meropol NJ, Schrag D, Smith TJ, et al: American Society of Clinical Oncology guidance statement: The cost of cancer care. J Clin Oncol 27:3868-3874, 2009

115. Fuchs V: What Factors Affect Health Care Expenditures and Health? Princeton, NJ: Robert Wood Johnson Foundation, 2011

116. Evangelista L, Bonavina MG, Bombardieri E: Clinical results and economic considerations of ${ }^{68} \mathrm{Ga}-\mathrm{PSMA}$ and radiolabeled choline in prostate cancer. Nucl Med Biol 50:47-49, 2017

117. Miles KA: Cancer imaging: Is it cost-effective? Cancer Imaging 4:97-103, 2004

118. Barlev A, Song X, Ivanov B, et al: Payer costs for inpatient treatment of pathologic fracture, surgery to bone, and spinal cord compression among patients with multiple myeloma or bone metastasis secondary to prostate or breast cancer. J Manag Care Pharm 16:693-702, 2010

119. Guttilla A, Bortolami A, Evangelista L: Prostate cancer as a chronic disease: Cost-effectiveness and proper follow-up. Q J Nucl Med Mol Imaging 59:439-445, 2015

120. Zeliadt SB, Penson DF: Pharmacoeconomics of available treatment options for metastatic prostate cancer. Pharmacoeconomics 25:309-327, 2007

121. Ferrell BR, Temel JS, Temin S, et al: Integration of palliative care into standard oncology care: American Society of Clinical Oncology clinical practice guideline update. J Clin Oncol 35:96-112, 2017

122. Morgan SC, Hoffman K, Loblaw DA, et al: Hypofractionated radiation therapy for localized prostate cancer: An ASTRO, ASCO, and AUA evidence-based guideline. J Clin Oncol 36:3411-3430, 2018

123. Bekelman JE, Rumble RB, Chen RC, et al: Clinically localized prostate cancer: ASCO Clinical Practice guideline endorsement of an American Urological Association/American Society for Radiation Oncology/Society of Urologic oncology guideline. J Clin Oncol 36:3251-3258, 2018

124. Morris MJ, Rumble RB, Basch E, et al: Optimizing anticancer therapy in metastatic non-castrate prostate cancer: American Society of Clinical Oncology clinical practice guideline. J Clin Oncol 36:1521-1539, 2018 


\section{Optimum Imaging Strategies for Advanced Prostate Cancer: ASCO Guideline}

The following represents disclosure information provided by authors of this manuscript. All relationships are considered compensated unless otherwise noted. Relationships are self-held unless noted. I = Immediate Family Member, Inst = My Institution. Relationships may not relate to the subject matter of this manuscript. For more information about ASCO's conflict of interest policy, please refer to www.asco.org/rwc or ascopubs.org/jco/site/ifc.

Open Payments is a public database containing information reported by companies about payments made to US-licensed physicians (Open Payments).

Edouard J. Trabulsi

Consulting or Advisory Role: GenomeDx

Speakers' Bureau: Johnson \& Johnson, Janssen Pharmaceuticals, Astellas Pharma, Medivation, Pfizer

\section{R. Bryan Rumble}

Employment: Park Lane Terrace (I)

Hossein Jadvar

Research Funding: Subtle Medical (Inst)

Thomas Hope

Honoraria: GE Healthcare

Consulting or Advisory Role: Ipsen, Curium

Research Funding: GE Healthcare, Philips Healthcare, Advanced Accelerator Applications (Inst)

Travel, Accommodations, Expenses: GE Healthcare

Martin Pomper

Employment: Johns Hopkins University

Leadership: Cancer Targeting Systems

Stock and Other Ownership Interests: Cancer Targeting Systems

Consulting or Advisory Role: Cancer Targeting Systems

Research Funding: Cancer Targeting Systems, Progenics Pharmaceuticals

Patents, Royalties, Other Intellectual Property: Patents licensed to Cance Targeting Systems, Progenics Pharmaceuticals, and Advanced Accelerator Applications (Inst); Neuraly (Inst); Theraly (Inst); Precision Molecular (Inst), FutureChem USA

\section{Baris Turkbey}

Patents, Royalties, Other Intellectual Property: Royalties from US government patents for magnetic resonance imaging-ultrasound fusion biopsy, computeraided diagnosis software

Other Relationship: Navidea, Philips Healthcare

Andrew B. Rosenkrantz

Patents, Royalties, Other Intellectual Property: Thieme Medical Publishers

Sadhna Verma

Honoraria: Hitachi (Ultrasound Division)

Speakers' Bureau: United Medical Systems

Travel, Accommodations, Expenses: Hitachi (Ultrasound Division), United Medical Systems

Daniel J. Margolis

Consulting or Advisory Role: Blue Earth Diagnostics

Research Funding: Siemens Healthineers (Inst)

Aytekin Oto

Honoraria: Bracco Diagnostics

Consulting or Advisory Role: Profound Healthcare, AbbVie

Research Funding: Philips Healthcare, Profound Healthcare, Guerbet

Travel, Accommodations, Expenses: Bracco Diagnostics

Andrei Purysko

Research Funding: Profound Medical (Inst), Invivo (Inst), Philips

Healthcare, RSNA

Travel, Accommodations, Expenses: Profound Healthcare

Matthew I. Milowsky

Consulting or Advisory Role: BioClin Therapeutics

Research Funding: Merck (Inst), Acerta Pharma (Inst), Roche (Inst), Genentech (Inst), Bristol-Myers Squibb (Inst), Seattle Genetics (Inst), Astellas Pharma (Inst) Clovis Oncology (Inst), Inovio Pharmaceuticals (Inst), AstraZeneca (Inst), X4

Pharmaceuticals (Inst), Mirati Therapeutics (Inst), Boehringer Ingelheim (Inst), Constellation Pharmaceuticals (Inst), Jounce Therapeutics (Inst), Syndax (Inst), Innocrin Pharma (Inst), MedImmune (Inst), Cerulean Pharma (Inst) Other Relationship: Asieris

Heinz-Peter Schlemmer

Honoraria: Bayer AG, Vital, Siemens Healthineers, Bracco Diagnostics, Curagita Consulting or Advisory Role: Siemens Healthineers, Bracco Diagnostics Research Funding: Siemens Healthineers (Inst), Profound Healthcare (Inst) Travel, Accommodations, Expenses: Siemens Healthineers, Bayer AG, Vital, Curagita, Bracco Diagnostics
Matthias Eiber

Consulting or Advisory Role: Blue Earth Diagnostics, ABX Advanced biochemical compounds

Research Funding: Siemens, Blue Earth Diagnostics, ABX Advanced biochemical compounds

Patents, Royalties, Other Intellectual Property: Patent application for radiohybrid prostate-specific membrane antigen

Travel, Accommodations, Expenses: Bayer Schering Pharma

Michael J. Morris

Consulting or Advisory Role: Astellas Pharma, Bayer AG, Endocyte, Advanced Accelerator Applications, Blue Earth Diagnostics, Tokai Pharmaceuticals, Tolmar Pharmaceuticals, ORIC Pharmaceuticals, Johnson \& Johnson Research Funding: Bayer AG (Inst), Sanofi (Inst), Endocyte (Inst), Progenics (Inst), Corcept Therapeutics (Inst), Roche (Inst), Genentech (Inst), Janssen Pharmaceuticals (Inst),

Travel, Accommodations, Expenses: Bayer AG, Endocyte, Fujifilm

Peter L. Choyke

Patents, Royalties, Other Intellectual Property: Patent holder for MRI-

ultrasound fusion technology licensed to Invivo, which markets it as UroNav; as a government employee, however, does not personally financial benefit from this patent

Other Relationship: Aspyrian Therapeutics, Philips Healthcare, GE Healthcare

Anwar Padhan

Honoraria: Janssen Oncology, Astellas Scientific and Medical Affairs Siemens Healthineers

Travel, Accommodations, Expenses: Siemens Healthineers

Jorge OIdan

Honoraria: Wolters Kluwer

Stefano Fanti

Honoraria: Bayer AG, Blue Earth Diagnostics, Sanofi, Astellas Pharma, Janssen Pharmaceuticals

Research Funding: Blue Earth Diagnostics

Travel, Accommodations, Expenses: Bayer AG, Blue Earth Diagnostics, Sanofi, Astellas Pharma

\section{Suneil Jain}

Consulting or Advisory Role: Janssen-Cilag, Movember, Bayer AG, Astellas

Pharma, Boston Scientific

Speakers' Bureau: Janssen-Cilag, Augmenix

Travel, Accommodations, Expenses: Janssen-Cilag, Astellas Pharma, Bayer AG

Peter A. Pinto

Patents, Royalties, Other Intellectual Property: National Institutes of Health $(\mathrm{NIH})$ and Philips have a cooperative research and development agreement. $\mathrm{NIH}$ has intellectual property in the field, including, among other patents and patent applications, "System, methods, and instrumentation for image guided prostate treatment," US patent number 8948845, with inventors/authors Brad Wood and Peter A. Pinto. NIH and Philips (Invivo) have a licensing agreement. $\mathrm{NIH}$ and authors receive royalties for a licensing agreement with Philips/Invivo. $\mathrm{NIH}$ does not endorse or recommend any commercial products, processes, or services. The views and personal opinions of authors expressed herein do not necessarily state or reflect those of the US government, nor reflect any official recommendation or opinion of the $\mathrm{NIH}$ or National Cancer Institute.

\section{Kirk A. Keegan}

Travel, Accommodations, Expenses: Intuitive Surgical, Taris BioMedical

\section{Christopher R. Porter}

Speakers' Bureau: Genomic Health

\section{Jonathan A. Coleman}

Travel, Accommodations, Expenses: Digital Angiography Reading Center (I) Other Relationship: Steba Biotech

Ashesh B. Jan

Consulting or Advisory Role: Blue Earth Diagnostics

No other potential conflicts of interest were reported. 


\section{APPENDIX}

TABLE A1. Optimum Imaging Strategies for Advanced Prostate Cancer Expert Panel Membership Name (and designation)

\begin{tabular}{|c|c|c|}
\hline Edouard J. Trabulsi, MD (co-chair) & $\begin{array}{l}\text { Sidney Kimmel Medical College at Thomas Jefferson University, } \\
\text { Philadelphia, PA }\end{array}$ & Urology \\
\hline H. Alberto Vargas, MD (co-chair) & Memorial Sloan Kettering Cancer Center, New York, NY & Radiology \\
\hline Jonathan A. Coleman, MD & Memorial Sloan Kettering Cancer Center, New York, NY & Urology \\
\hline Peter L. Choyke, MD & National Cancer Institute, Bethesda, MD & Radiology \\
\hline Stefano Fanti, MD & University of Bologna, Bologna, Italy & Nuclear medicine \\
\hline Adam Froemming, MD & Mayo Clinic, Rochester, MN & Radiology \\
\hline Thomas Hope, MD & University of California, San Francisco, San Francisco CA & $\begin{array}{l}\text { Abdominal imaging and nuclear } \\
\text { medicine }\end{array}$ \\
\hline Kirk A. Keegan, MD & Vanderbilt Urologic Surgery, Nashville, TN & Urology \\
\hline Jeffrey M. Kamradt, MD & Hartford Hospital, Hartford, CT & PGIN \\
\hline Daniel J. Margolis, MD & Weill Cornell Medical College, New York City, NY & Radiology \\
\hline Matthew I. Milowsky, MD & UNC Lineberger Comprehensive Cancer Center, Chapel Hill, NC & Medical oncology \\
\hline Michael J. Morris, MD & Memorial Sloan Kettering Cancer Center, New York, NY & Medical oncology \\
\hline Jorge Oldan, MD & UNC Lineberger Comprehensive Cancer Center, Chapel Hill, NC & Nuclear medicine \\
\hline Aytekin Oto, MD & The University of Chicago, Chicago, IL & Radiology \\
\hline Anwar Padhani, MD & $\begin{array}{l}\text { Paul Strickland Scanner Centre, Mount Vernon Cancer Centre, } \\
\text { Northwood, United Kingdom }\end{array}$ & Radiology \\
\hline Andrew B. Rosenkrantz, MD & NYU Langone Health, New York, NY & Radiology \\
\hline Westley Sholes, MPA & & Patient representative \\
\hline Baris Turkbey, MD & National Cancer Institute, Bethesda, MD & Radiology \\
\hline Sadhna Verma, MD & University of Cincinnati Medical Center, Cincinnati, $\mathrm{OH}$ & Radiology \\
\hline R. Bryan Rumble, MSc & American Society of Clinical Oncology & Staff/health research meth \\
\hline
\end{tabular}

Abbreviation: PGIN, Practice Guideline Information Network. 